\title{
Enhanced mixing of intrinsic states in deformed Hf nuclei
}

\author{
E. A. McCutchan, ${ }^{1,2}$ R. F. Casten, ${ }^{2}$ V. Werner, ${ }^{2}$ E. Williams, ${ }^{2}$ R. Winkler, ${ }^{2}$ A. Wolf,,${ }^{2,3}$ Z. Berant, ${ }^{2,3}$ G. Gürdal, ${ }^{2,4}$ J. Qian, ${ }^{2}$ \\ H. Ai, ${ }^{2}$ L. Amon, ${ }^{5}$ C. W. Beausang, ${ }^{6}$ D. S. Brenner, ${ }^{4}$ R. B. Cakirli, ${ }^{5}$ R. J. Casperson, ${ }^{2}$ C. R. Fitzpatrick, ${ }^{2,7}$ D. Frank, ${ }^{2}$ \\ A. B. Garnsworthy,,$^{2,7}$ A. Heinz, ${ }^{2}$ R. Lüttke, ${ }^{2,8}$ A. F. Mertz, ${ }^{2}$ Y. Oktem, ${ }^{5}$ N. Pietralla, ${ }^{8}$ P. H. Regan, ${ }^{7}$ B. Shoraka, ${ }^{2,7}$ \\ J. R. Terry, ${ }^{2}$ and N. J. Thompson ${ }^{2,7}$ \\ ${ }^{1}$ Physics Division, Argonne National Laboratory, Argonne, Illinois 60439, USA \\ ${ }^{2}$ Wright Nuclear Structure Laboratory, Yale University, New Haven, Connecticut 06520, USA \\ ${ }^{3}$ Nuclear Research Center Negev, Beer-Sheva 84190, Israel \\ ${ }^{4}$ Chemistry Department, Clark University, Worcester, Massachusetts 01610, USA \\ ${ }^{5}$ Department of Physics, University of Istanbul, Istanbul, Turkey \\ ${ }^{6}$ University of Richmond, Richmond, Virginia, USA \\ ${ }^{7}$ Department of Physics, University of Surrey, Guildford, Surrey GU2 7XH, United Kingdom \\ ${ }^{8}$ Technische Universität Darmstadt, D-64289 Darmstadt, Germany
}

(Received 19 February 2008; published 2 May 2008)

\begin{abstract}
Excited low-spin, nonyrast states in ${ }^{170,172,174} \mathrm{Hf}$ were populated in $\beta^{+} / \epsilon$ decay and studied through off-beam $\gamma$-ray spectroscopy. New coincidence data allowed for a substantial revision of the level schemes of ${ }^{170,172} \mathrm{Hf}$ and a confirmation of the level scheme of ${ }^{174} \mathrm{Hf}$. The $\mathrm{Hf}$ isotopes represent a unique situation in which a crossing of collective intrinsic excitations occurs, enhancing significantly the effects of mixing. Using branching ratios from excited $2^{+}$states, this mixing is followed and studied. The resulting mixing matrix elements are found to be $\sim 30 \mathrm{keV}$ - an order of magnitude larger than estimated previously for nearby nuclei. In the case of ${ }^{170} \mathrm{Hf}$, the $2_{\beta}^{+}$ and $2_{\gamma}^{+}$level are shown to be completely mixed.
\end{abstract}

DOI: 10.1103/PhysRevC.77.054304

PACS number(s): 21.10.Re, 21.60.Ev, 23.20.Lv, 27.70.+q

\section{INTRODUCTION}

The deformed axially symmetric rotor [1] is described by a nonzero value of the quadrupole deformation, $\beta_{o}$ and $\gamma$ fixed at $0^{\circ}$. When quadratic small amplitude oscillations in $\beta$ and $\gamma$ are allowed, then additional collective excitations can occur. The most common of these are oscillations along the symmetry axis (so called $\beta$ vibrations) or oscillations perpendicular to the symmetry axis ( $\gamma$ vibrations). The term " $\beta$ " vibration is used here only as a label for the first excited $0^{+}$excitation, because, microscopically, such excitations are forbidden within a major shell [1]. The wave functions for these states can be separated into the product of the intrinsic excitations and the rotational motion. For $B(E 2)$ branching ratios between bands, the intrinsic matrix elements cancel; the ratio then depends only on the ratio of the squares of Clebsch-Gordan coefficients, as given by the well-known Alaga rules [2].

Very early on, simple predictions of the Alaga rules were observed to be unfulfilled in most well-deformed nuclei, due to a (small) breakdown of the adiabatic assumption. A traditional way of treating this nonadiabatic coupling is to consider bandmixing [3] among the ground, $\beta$, and $\gamma$ bands. Usually, bandmixing is limited to a simple analysis in terms of $\Delta K=2$ mixing between the $\gamma$ band and ground band. Attempts at involving the mixing of the $\beta$ and $\gamma$ bands have been met with limited success (see, for example, Ref. [4-6]).

One area in which the mixing between the $\gamma$ and $\beta$ vibrational states has been studied in great detail is the tungsten isotopes. Initial studies [7,8] involving a variety of different models indicated a small mixing between the $\gamma$ and $\beta$ bands. In addition, later calculations, based on improved measurements of $B(E 2)$ transition strengths, yielded absolute matrix elements between the $2_{\gamma}^{+}$and $2_{\beta}^{+}$states in ${ }^{182} \mathrm{~W}$ on the order of $\sim 2 \mathrm{keV}[9,10]$.

The Hf isotopic chain provides an additional interesting region to study the evolution of two-level mixing among excited $2^{+}$states belonging to the $\gamma$ and $\beta$ bands. The well-deformed $\mathrm{Hf}$ nuclei (in particular ${ }^{172} \mathrm{Hf}$ ) are known to possess a set of high $K$ isomers [11-13]. This gives good support for axial symmetry in these nuclei and $K$, the projection of angular momentum onto the symmetry axis, can be considered an approximately good quantum number. Furthermore, the energies of the $2_{\gamma}^{+}$and $2_{\beta}^{+}$levels in ${ }^{166} \mathrm{Hf}$ through ${ }^{174} \mathrm{Hf}$ [14-18] follow interesting trajectories, as shown in Fig. 1. These systematics show a crossing of the $\beta$ and $\gamma$ modes and represent a unique situation in which the strength of the interaction between the $2_{\gamma}^{+}$and $2_{\beta}^{+}$states can be investigated as a function of the energy separation between the two levels, with a near degeneracy precisely at ${ }^{170} \mathrm{Hf}$. Interactions between quadrupole intrinsic excitations are expected to be large near an (avoided) crossing, as studied in Ref. [19].

The purpose of this study is to measure the energies and decay properties of low-lying levels in ${ }^{170,172,174} \mathrm{Hf}$ populated in $\beta^{+} / \epsilon$ decay. The present work makes use of high-statistics coincidence data, leading to a substantial revision of the previous ${ }^{170,172} \mathrm{Hf}$ level schemes and improved measurements of intensities of low-lying transitions. The level scheme of ${ }^{174} \mathrm{Hf}$ was confirmed, with a few additional transitions identified. With sensitive $\gamma$-ray spectroscopy and improved measurements of $\gamma$-ray transition intensities, the abovementioned interactions can be studied around the crossing region using the high sensitivity of relative $B(E 2)$ values to variations in the mixing. 


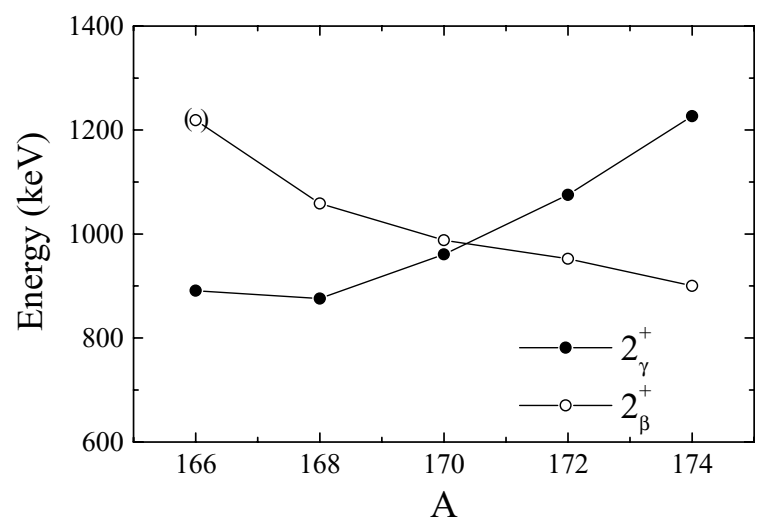

FIG. 1. Energy systematics of the $2_{\gamma}^{+}$and $2_{\beta}^{+}$levels in the Hf isotopes. The parenthesis for the $2_{\beta}^{+}$level in ${ }^{166} \mathrm{Hf}$ indicates a tentative assignment. Band assignments taken from Refs. [14-18].

\section{II. $\beta$-DECAY SPECTROSCOPY EXPERIMENTS}

Low-spin states in ${ }^{170} \mathrm{Hf}$ were populated in the $\beta^{+} / \epsilon$ decay of ${ }^{170} \mathrm{Ta}$ and studied through off-beam $\gamma$-ray spectroscopy. The parent ${ }^{170} \mathrm{Ta}$ nuclei were produced through the ${ }^{159} \mathrm{~Tb}\left({ }^{16} \mathrm{O}\right.$, $5 n$ ) reaction by bombarding a $4.4-\mathrm{mg} / \mathrm{cm}^{2}$ target with a $\sim 14 \mathrm{pnA}, 100-\mathrm{MeV}{ }^{16} \mathrm{O}$ beam provided by the Yale ESTU tandem accelerator. The reaction products are emitted in a forward solid angle cone, deposited on a 16-mm-wide aluminized Kapton tape and transported periodically to the center of a superconducting coil. To prevent the primary beam from reaching the tape, a 3-mm-diameter gold plug was placed between the target and the tape. The tape was moved cyclically every $15 \mathrm{~min}$ to optimize the observed decay from the parent ${ }^{170} \mathrm{Ta}\left(T_{1 / 2}=6.8 \mathrm{~min}\right)$ nuclei. ${ }^{170} \mathrm{Ta}$ decays to ${ }^{170} \mathrm{Hf}$ with a $Q$ value of $6.1 \mathrm{MeV}$ [16].

The $\gamma$ rays were detected by a set of eight coaxial HPGe detectors positioned around the center of the coil. $\gamma-\gamma$ coincidence data were acquired in event mode with a doubles trigger. In the $\sim 200$-h experiment, $1.3 \times 10^{9} \gamma-\gamma$ coincidence events were acquired. The original aim of this experiment on ${ }^{170} \mathrm{Hf}$ was a measurement of the $g$ factor of the $2_{1}^{+}$state. This result has been published [20] and here we report on the new $\gamma-\gamma$ coincidence data obtained from that experiment.

Low-spin states in ${ }^{172,174} \mathrm{Hf}$ were populated in the $\beta^{+} / \epsilon$ decay of ${ }^{172,174} \mathrm{Ta}$ and studied through off-beam $\gamma$-ray spectroscopy. The parent ${ }^{172} \mathrm{Ta}$ nuclei were produced through the ${ }^{165} \mathrm{Ho}\left({ }^{12} \mathrm{C}, 5 n\right)$ reaction by bombarding a $\sim 4.0-\mathrm{mg} / \mathrm{cm}^{2}$ target with a $\sim 2 \mathrm{pnA}, 80-\mathrm{MeV}{ }^{12} \mathrm{C}$ beam. The parent ${ }^{174} \mathrm{Ta}$ nuclei were produced through the ${ }^{168} \operatorname{Er}\left({ }^{11} \mathrm{~B}, 5 n\right)$ reaction by bombarding a $3.5-\mathrm{mg} / \mathrm{cm}^{2}$ target with a $\sim 3 \mathrm{pnA}, 65-\mathrm{MeV}^{11} \mathrm{~B}$ beam. Both beams were provided by the Yale ESTU tandem accelerator. The ${ }^{172} \mathrm{Hf}\left({ }^{174} \mathrm{Hf}\right)$ experiment was performed with a beam-on/beam-off cycle of 120 (200) sec intervals. The $T_{1 / 2}$ for ${ }^{172} \mathrm{Ta}$ decay is $36.8 \mathrm{~min}$, with a $Q$ value of $4.9 \mathrm{MeV}$ [17] to ${ }^{172} \mathrm{Hf}$. The $T_{1 / 2}$ for ${ }^{174} \mathrm{Ta}$ decay is $1.14 \mathrm{~h}$, with a $Q$ value of $3.8 \mathrm{MeV}[18]$ to ${ }^{174} \mathrm{Hf}$.

During the beam-off cycle, $\gamma$ rays were detected using eight Compton-suppressed segmented YRAST ball clover HPGe detectors [21]. Both $\gamma$-ray singles and $\gamma-\gamma$ coincidence data were acquired in event mode. The ${ }^{172} \mathrm{Hf}$ experiment yielded
$2.6 \times 10^{7}$ clover-clover coincidence events and $2.7 \times 10^{7}$ clover singles events. The ${ }^{174} \mathrm{Hf}$ experiment yielded $8.4 \times 10^{7}$ clover-clover coincidence events and $6.3 \times 10^{7}$ clover singles events.

\section{A. Levels in ${ }^{170} \mathrm{Hf}$}

The level scheme obtained in the present work provided substantial modification to the current literature $\beta^{+} / \epsilon$ decay data [16] on ${ }^{170} \mathrm{Hf}$. The evaluated $\beta$-decay data are based on a single previous $\beta$-decay measurement [22]. Here, nine new levels are identified, no evidence is found for one previous proposed level, and the decay properties of some of the remaining levels are substantially modified. Table I lists the levels populated in ${ }^{170} \mathrm{Hf}$ and their $\gamma$ decay. Because data were collected with only a doubles trigger, the intensity of the $101-\mathrm{keV}, 2_{1}^{+} \rightarrow 0_{1}^{+}$transition could not be determined. In the following discussion, intensities are normalized to the intensity of the $221-\mathrm{keV}, 4_{1}^{+} \rightarrow 2_{1}^{+}$transition $\left(I_{221} \equiv 100\right)$. The complete level scheme deduced in the present work is given in Fig. 2. Spin and parity assignments are taken from the literature [16] except where noted.

\section{1. $\left(2^{+}\right)$level at $988 \mathrm{keV}$}

This level was previously reported [16] to decay to the $4_{1}^{+}, 2_{1}^{+}$, and $0_{1}^{+}$levels through transitions of 666,887 , and $988 \mathrm{keV}$, respectively. In the present work, a 988.04(6)-keV transition to the $0_{1}^{+}$level and a $666.35(8)-\mathrm{keV}$ transition to the $4_{1}^{+}$level are observed with intensities similar to those given in the literature. No evidence is found for a $887-\mathrm{keV}$ transition to the $2_{1}^{+}$level. As illustrated in Fig. 3(a), a gate on the $101-\mathrm{keV}, 2_{1}^{+} \rightarrow 0_{1}^{+}$transition finds no evidence for a $887-\mathrm{keV}$ transition with reported [16] intensity of 2.4(5). For comparison, the $780-\mathrm{keV}$ transition [with a reported intensity of 5.8(4)], which directly feeds the $2_{1}^{+}$level, is highlighted in Fig. 3(a). Figure 3(b) shows a summed spectrum gated on the $437-\mathrm{keV}$ and $710-\mathrm{keV}$ transitions that populate the level at $988 \mathrm{keV}$. Strong coincidences are observed with the 666and $988-\mathrm{keV}$ depopulating transitions; however, no evidence is found for a $887-\mathrm{keV}$ depopulating transition. In the present work, we obtain a limit of $<1.0$ for the intensity of a $887-\mathrm{keV}$ transition to the $2_{1}^{+}$level. The observed transitions to the $0^{+}$ and $4^{+}$states are still consistent with a $2^{+}$spin assignment for this level.

\section{2. $\left(4^{+}\right)$level at $1155 \mathrm{keV}$}

This level was proposed in $\beta$ decay [22] on the basis of three depopulating transitions, a $1054-\mathrm{keV}$ transition to the $2_{1}^{+}$level, a $835-\mathrm{keV}$ transition to the $4_{1}^{+}$level, and a tentatively placed transition of $512.5-\mathrm{keV}$ to the $6_{1}^{+}$level. In the present work, we identify a level at $1158.95(8) \mathrm{keV}$ that we associate with the reported $1155-\mathrm{keV}$ level. This identification is based on two depopulating transitions. A 837.16(7)-keV transition is found to be coincident with the $221-\mathrm{keV}, 4_{1}^{+} \rightarrow 2_{1}^{+}$transition, as shown in Fig. 4, and placed as directly populating the $4_{1}^{+}$level. A 516.3(2)-keV transition is found to be coincident with the 321-keV, $6_{1}^{+} \rightarrow 4_{1}^{+}$transition, as shown in Fig. 5, and placed as directly populating the $6_{1}^{+}$level. We find no evidence for 
TABLE I. Levels populated in ${ }^{170} \mathrm{Hf}$ and their $\gamma$ decay. Relative intensities (in $\beta$ decay) are given for $\gamma$-ray transitions depopulating the levels and compared with literature values [16] where available.

\begin{tabular}{|c|c|c|c|c|c|c|c|c|}
\hline$J_{i}^{\pi}$ & $E_{i}(\mathrm{keV})$ & $J_{f}^{\pi}$ & $E_{f}(\mathrm{keV})$ & $E_{\gamma}(\mathrm{keV})$ & $I_{\gamma}$ & $I_{\text {lit }}{ }^{\mathrm{a}}$ & $I_{\gamma}^{\text {rel }}$ & $I_{\mathrm{lit}}^{\mathrm{rel}}$ \\
\hline $2^{+}$ & $100.75(4)$ & $0^{+}$ & 0.00 & $100.75(4)$ & & & & \\
\hline $4^{+}$ & $321.80(6)$ & $2^{+}$ & 100.75 & 221.05(5) & $100(2)$ & $100(3)$ & $100(2)$ & $100(3)$ \\
\hline $6^{+}$ & $642.59(6)$ & $4^{+}$ & 321.80 & $320.79(7)$ & $5.3(2)$ & $4.4(3)$ & $100(4)$ & $100(7)$ \\
\hline $0^{+}$ & $880.33(7)$ & $2^{+}$ & 100.75 & $779.58(6)$ & $8.0(4)$ & $5.8(4)$ & $100(5)$ & $100(7)$ \\
\hline \multirow[t]{3}{*}{$2^{+}$} & $961.96(7)$ & $0^{+}$ & 0.00 & $961.95(7)$ & $2.4(2)$ & $2.9(12)$ & $5.5(5)$ & $6.2(26)$ \\
\hline & & $2^{+}$ & 100.75 & $861.18(6)$ & 43.7(13) & $47.1(16)$ & $100(3)$ & $100(3)$ \\
\hline & & $4^{+}$ & 321.80 & $640.19(9)$ & 4.3(3) & $3.1(4)$ & $9.8(7)$ & $6.6(9)$ \\
\hline \multirow[t]{3}{*}{$2^{+}$} & $988.06(8)$ & $0^{+}$ & 0.00 & $988.04(6)$ & $46(3)$ & $37(2)$ & $100(6)$ & $100(5)$ \\
\hline & & $2^{+}$ & 100.75 & [887] & $<1.0$ & $2.4(5)$ & $<2$ & $6.5(14)$ \\
\hline & & $4^{+}$ & 321.80 & $666.35(8)$ & 11.2(9) & $7.1(8)$ & $24.3(20)$ & $19.2(22)$ \\
\hline \multirow[t]{2}{*}{$3^{+}$} & $1087.85(9)$ & $2^{+}$ & 100.75 & $987.04(5)$ & $23(1)$ & 21(1) & $100(4)$ & $100(5)$ \\
\hline & & $4^{+}$ & 321.80 & $766.13(7)$ & $6.9(4)$ & $6.2(9)$ & $30(2)$ & $30(4)$ \\
\hline \multirow[t]{3}{*}{$4^{+}$} & $1158.95(8)^{\mathrm{b}}$ & $2^{+}$ & 100.75 & [1058] & $<0.70$ & $1.9(4)$ & $<6$ & $19(4)$ \\
\hline & & $4^{+}$ & 321.80 & $837.16(7)^{\mathrm{c}}$ & $12.3(8)$ & $9.8(8)$ & $100(7)$ & $100(8)$ \\
\hline & & $6^{+}$ & 642.59 & $516.3(2)^{c}$ & $0.81(27)$ & & $6.6(22)$ & \\
\hline \multirow[t]{3}{*}{$4^{+}$} & $1227.55(8)$ & $2^{+}$ & 100.75 & $1126.7(1)$ & $7.0(4)$ & $3.2(3)$ & $100(6)$ & $100(9)$ \\
\hline & & $4^{+}$ & 321.80 & $905.66(6)$ & $3.2(3)$ & $2.5(4)$ & $46(4)$ & $78(13)$ \\
\hline & & $6^{+}$ & 642.59 & $585.04(8)$ & $0.78(9)$ & $2.1(3)$ & $11(1)$ & $66(9)$ \\
\hline \multirow[t]{29}{*}{$\left(5^{-}\right)$} & $1372.82(10)^{\mathrm{d}}$ & $4^{+}$ & 321.80 & $1051.02(10)^{\mathrm{e}}$ & $2.8(2)$ & & $100(7)$ & \\
\hline & & $6^{+}$ & 642.59 & $730.2(1)^{\mathrm{e}}$ & $0.50(7)$ & & $18(3)$ & \\
\hline & $1425.31(10)^{\mathrm{f}}$ & $2^{+}$ & 100.75 & $1324.5(2)^{\mathrm{g}}$ & $2.3(3)$ & & $15(2)$ & \\
\hline & & $2^{+}$ & 961.96 & $463.37(8)^{\mathrm{g}}$ & $15.2(20)$ & & $100(13)$ & \\
\hline & & $2^{+}$ & 988.06 & $437.18(9)^{\mathrm{g}}$ & $5.4(4)$ & & $36(3)$ & \\
\hline & & $3^{+}$ & 1087.85 & $337.53(10)^{\mathrm{g}}$ & $5.3(4)$ & & $35(3)$ & \\
\hline & $1441.71(7)^{b}$ & $2^{+}$ & 100.75 & $1340.97(8)^{\mathrm{c}}$ & $8.2(5)$ & $9.1(7)$ & $83(5)$ & $128(10)$ \\
\hline & & $4^{+}$ & 321.80 & $1119.91(8)^{\mathrm{h}}$ & $9.9(7)$ & $7.1(5)$ & $100(7)$ & $100(7)$ \\
\hline & $1563.98(11)^{\mathrm{f}}$ & $4^{+}$ & 321.80 & $1242.8(2)^{\mathrm{g}}$ & $0.82(18)$ & & $23(5)$ & \\
\hline & & $3^{+}$ & 1087.85 & $476.08(7)^{\mathrm{g}}$ & $3.6(4)$ & & $100(11)$ & \\
\hline & & $4^{+}$ & 1158.95 & $405.06(8)^{\mathrm{g}}$ & $0.94(11)$ & & $26(3)$ & \\
\hline & & $\left(5^{-}\right)$ & 1372.82 & $191.3(2)^{\mathrm{g}}$ & $0.42(11)$ & & $12(3)$ & \\
\hline & $1565.65(10)^{\mathrm{f}}$ & $4^{+}$ & 321.80 & $1243.8(1)^{\mathrm{g}}$ & $1.3(3)$ & & $100(23)$ & \\
\hline & & $6^{+}$ & 642.59 & $923.1(1)^{g}$ & $0.78(8)$ & & $60(6)$ & \\
\hline & $1573.20(15)^{\mathrm{f}}$ & $2^{+}$ & 100.75 & $1476.5(2)^{\mathrm{g}}$ & $8.9(7)$ & & $100(8)$ & \\
\hline & & $4^{+}$ & 321.80 & $1251.35(9)^{\mathrm{g}}$ & $5.7(4)$ & & $64(5)$ & \\
\hline & $1583.38(11)^{\mathrm{f}}$ & $2^{+}$ & 100.75 & $1482.64(9)^{\mathrm{g}}$ & $1.8(4)$ & & $43(10)$ & \\
\hline & & $0^{+}$ & 880.33 & $703.2(2)^{\mathrm{g}}$ & $1.05(8)$ & & $26(2)$ & \\
\hline & & $2^{+}$ & 961.96 & $621.3(1)^{\mathrm{g}}$ & $0.38(4)$ & & $9.5(10)$ & \\
\hline & & $2^{+}$ & 988.06 & $595.26(7)^{\mathrm{g}}$ & $4.0(5)$ & & $100(13)$ & \\
\hline & $1658.8(1)^{\mathrm{f}}$ & $2^{+}$ & 100.75 & $1558.0(1)^{\mathrm{g}}$ & $4.5(3)$ & & $100(7)$ & \\
\hline & & $4^{+}$ & 321.80 & $1337.05(9)^{\mathrm{g}}$ & $1.5(2)$ & & $33(4)$ & \\
\hline & $1697.9(1)^{\mathrm{f}}$ & $2^{+}$ & 961.96 & $735.88(10)^{\mathrm{g}}$ & $3.0(3)$ & & $70(7)$ & \\
\hline & & $2^{+}$ & 988.06 & $710.00(11)^{\mathrm{g}}$ & $4.3(3)$ & & $100(7)$ & \\
\hline & $1998.9(1)^{\mathrm{f}}$ & $2^{+}$ & 988.06 & $1010.9(1)^{\mathrm{g}}$ & $1.13(9)$ & & $88(7)$ & \\
\hline & & & 1425.31 & $573.59(8)^{\mathrm{g}}$ & $1.28(16)$ & & $100(13)$ & \\
\hline & $2117.2(1)^{\mathrm{f}}$ & $2^{+}$ & 961.96 & $1155.29(8)^{\mathrm{g}}$ & $3.2(3)$ & & $100(9)$ & \\
\hline & & $2^{+}$ & 988.06 & $1129.18(9)^{\mathrm{g}}$ & $2.0(3)$ & & $63(9)$ & \\
\hline & & $3^{+}$ & 1087.85 & $1029.38(8)^{\mathrm{g}}$ & $1.6(3)$ & & $50(9)$ & \\
\hline
\end{tabular}

${ }^{a}$ Literature values for intensities are from the evaluated ${ }^{170} \mathrm{Ta}$ EC data of Ref. [16].

${ }^{\mathrm{b}}$ Level reported in $\beta$ decay with 4-keV difference but similar decay transitions.

${ }^{\mathrm{c}}$ Transitions reported in $\beta$ decay but with $4-\mathrm{keV}$ difference.

${ }^{\mathrm{d}}$ Level was not previously reported in $\beta$ decay.

${ }^{\mathrm{e}} \gamma$-ray line was not previously reported in $\beta$ decay.

${ }^{\mathrm{f}}$ Level was not previously reported.

${ }^{\mathrm{g}} \gamma$-ray line was not previously reported.

${ }^{\mathrm{h}} \gamma$-ray line previously reported but in alternate placement. 


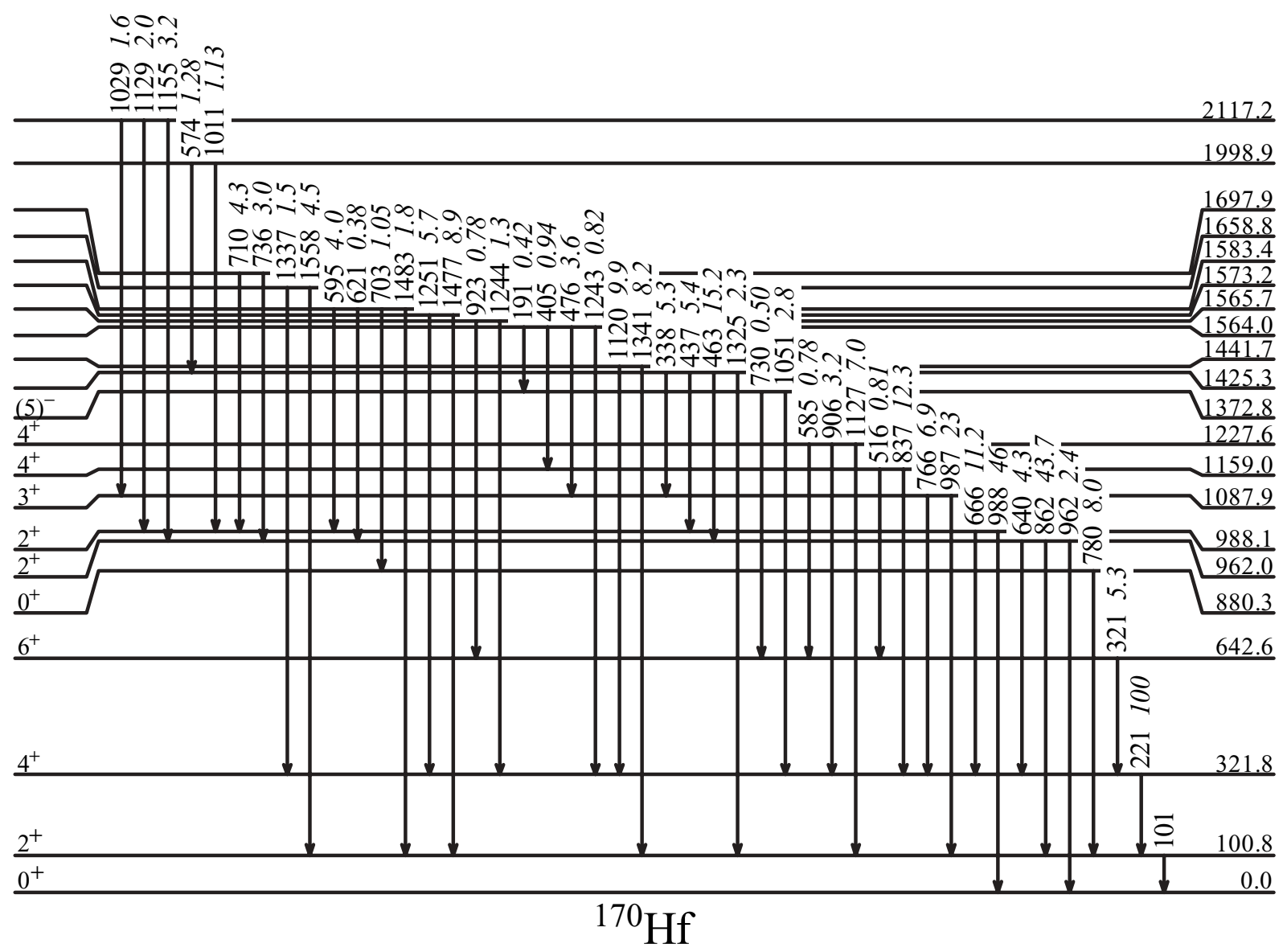

FIG. 2. Complete level scheme for ${ }^{170} \mathrm{Hf}$ populated in the $\beta^{+} / \epsilon$ decay of ${ }^{170} \mathrm{Ta}$. Levels are marked with their energy in keV. $\gamma$-ray transitions are labeled by their energy in $\mathrm{keV}$ and relative intensities (in italics), normalized to the $221-\mathrm{keV}, 4_{1}^{+} \rightarrow 2_{1}^{+} \operatorname{transition}\left(I_{221} \equiv 100\right)$.

a $1054-\mathrm{keV}$ (1058 keV according to our energy calibration) transition to the $2_{1}^{+}$level. As shown in Fig. 3(a), a gate on the $101-\mathrm{keV}, 2_{1}^{+} \rightarrow 0_{1}^{+}$transition gives no evidence for a 1058-keV transition with reported [16] intensity of 1.9(4). For comparison, the $1127-\mathrm{keV}$ transition [with a reported intensity of 3.2(3)], which directly feeds the $2_{1}^{+}$level, is highlighted in Fig. 3(a). In the present work, we obtain a limit of $<0.70$ for the intensity of a $1058-\mathrm{keV}$ transition to the $2_{1}^{+}$level.

\section{3. $\left(4^{+}\right)$level at $1219 \mathrm{keV}$}

This level was proposed [22] in $\beta$ decay on the basis of three depopulating transitions of 1120,898 , and $576.5 \mathrm{keV}$ to the $2_{1}^{+}, 4_{1}^{+}$, and $6_{1}^{+}$levels, respectively. The present experiment finds a $1119.91(8)-\mathrm{keV}$ transition in coincidence with the 221-keV, $4_{1}^{+} \rightarrow 2_{1}^{+}$transition, as shown in Fig. 6(a). Furthermore, the $1120-\mathrm{keV}$ transition is coincident with only the 101- and 221-keV transitions, as shown in Fig. 6(b). This provides an alternate placement for the $1120-\mathrm{keV}$ transition as depopulating a newly proposed level at $1442 \mathrm{keV}$ and directly feeding the $4_{1}^{+}$level. As shown in Fig. 4, a gate on the $221-\mathrm{keV}, 4_{1}^{+} \rightarrow 2_{1}^{+}$transition finds no coincidences with a transition of $898 \mathrm{keV}$, reported [16] with an intensity of $0.9(3)$. For comparison, the 906-keV transition highlighted in Fig. 4 does feed the $4_{1}^{+}$level with a reported intensity of 2.4(4). As shown in Fig. 5, a gate on the $321-\mathrm{keV}, 6_{1}^{+} \rightarrow 4_{1}^{+}$transition finds no coincidences with a 577-keV transition, reported with an intensity of 1.9(3). As a reference, the 585-keV transition highlighted in Fig. 5 does feed the $6_{1}^{+}$level with a reported intensity of 2.1(3). With no evidence for any of the proposed depopulating transitions, we eliminate the level at $1219 \mathrm{keV}$.

Nine new levels are identified in ${ }^{170} \mathrm{Hf}$ above $1400 \mathrm{keV}$ based on the observation of several depopulating transitions. These levels and their decay are summarized in Table I.

\section{B. Levels in ${ }^{172} \mathrm{Hf}$}

The level scheme obtained in the present work for ${ }^{172} \mathrm{Hf}$ is also a substantial revision of the current literature $\beta^{+} / \epsilon$ decay [17] data on ${ }^{172} \mathrm{Hf}$. The $\beta$-decay data of ${ }^{172} \mathrm{Hf}$ are based on a single previous $\beta$-decay study [23]. Here, 10 new levels are identified, no evidence is found for one previously proposed level, and many of the previously unplaced transitions are now given definite placements in the level scheme based on the new coincidence data. Table II lists the levels populated in ${ }^{172} \mathrm{Hf}$ and their $\gamma$ decay. In the following discussion, intensities are normalized to the intensity of the $214-\mathrm{keV}, 4_{1}^{+} \rightarrow 2_{1}^{+}$transition $\left(I_{214} \equiv 100\right)$. The ${ }^{172} \mathrm{Hf}$ level scheme for levels below $1500 \mathrm{keV}$ deduced in 
TABLE II. Levels populated in ${ }^{172} \mathrm{Hf}$ and their $\gamma$ decay. Relative intensities (in $\beta$ decay) are given for $\gamma$-ray transitions depopulating the levels and compared with literature values [17] where available.

\begin{tabular}{|c|c|c|c|c|c|c|c|c|}
\hline$J_{i}^{\pi}$ & $E_{i}(\mathrm{keV})$ & $J_{f}^{\pi}$ & $E_{f}(\mathrm{keV})$ & $E_{\gamma}(\mathrm{keV})$ & $I_{\gamma}$ & $I_{\text {lit }}{ }^{\mathrm{a}}$ & $I_{\gamma}^{\mathrm{rel}}$ & $I_{\text {lit }}^{\text {rel }}$ \\
\hline $2^{+}$ & $95.24(4)$ & $0^{+}$ & 0.00 & $95.24(4)$ & $40.0(20)$ & $38.7(15)$ & $100(5)$ & $100(4)$ \\
\hline $4^{+}$ & $309.31(6)$ & $2^{+}$ & 95.24 & 214.07(4) & $100(3)$ & 100 & $100(3)$ & 100 \\
\hline $6^{+}$ & $628.53(6)$ & $4^{+}$ & 309.31 & $319.22(6)$ & $8.94(20)$ & $9.54(18)$ & $100(3)$ & $100(2)$ \\
\hline $0^{+}$ & $871.45(6)$ & $2^{+}$ & 95.24 & $776.21(6)$ & $3.6(2)$ & $4.59(9)$ & $100(6)$ & $100(2)$ \\
\hline \multirow[t]{3}{*}{$2^{+}$} & $952.78(6)$ & $0^{+}$ & 0.00 & $952.78(7)$ & $3.1(1)$ & $3.55(15)$ & $48(2)$ & $44(2)$ \\
\hline & & $2^{+}$ & 95.24 & $857.46(5)$ & $6.4(2)$ & $8.0(3)$ & $100(3)$ & $100(4)$ \\
\hline & & $4^{+}$ & 309.31 & $643.48(8)$ & $3.3(1)$ & $4.15(16)$ & $52(2)$ & $52(2)$ \\
\hline \multirow[t]{3}{*}{$2^{+}$} & $1075.63(7)$ & $0^{+}$ & 0.00 & $1075.62(8)$ & $6.8(2)$ & $6.66(16)$ & $99(3)$ & $94(2)$ \\
\hline & & $2^{+}$ & 95.24 & $980.40(8)$ & $6.9(2)$ & $7.07(10)$ & $100(3)$ & $100(1)$ \\
\hline & & $4^{+}$ & 309.31 & $766.2(1)^{\mathrm{b}}$ & $0.4(1)$ & & $5.8(15)$ & \\
\hline \multirow[t]{4}{*}{$4^{+}$} & 1129.94(8) & $2^{+}$ & 95.24 & 1034.69(8) & $3.8(2)$ & $3.70(18)$ & $63(3)$ & $64(3)$ \\
\hline & & $4^{+}$ & 309.31 & $820.66(7)$ & $6.00(15)$ & $5.80(24)$ & $100(3)$ & $100(4)$ \\
\hline & & $6^{+}$ & 628.53 & $501.4(1)$ & $0.80(7)$ & $1.41(22)$ & $13(1)$ & $24(4)$ \\
\hline & & $2^{+}$ & 952.78 & $177.4(2)^{\mathrm{b}}$ & $0.12(2)$ & & $2.0(3)$ & \\
\hline \multirow[t]{2}{*}{$3^{+}$} & $1181.39(8)$ & $2^{+}$ & 95.24 & $1086.10(8)$ & $14.2(6)$ & $14.7(5)$ & $100(4)$ & $100(3)$ \\
\hline & & $4^{+}$ & 309.31 & 872.13(9) & $3.32(15)$ & $2.6(10)$ & $23(1)$ & $18(7)$ \\
\hline $0^{+}$ & $1294.9(2)$ & $2^{+}$ & 95.24 & 1197.2(1) & $0.17(4)$ & $0.54(8)$ & $100(24)$ & $100(15)$ \\
\hline \multirow[t]{3}{*}{$4^{+}$} & $1304.97(9)^{\mathrm{c}}$ & $2^{+}$ & 95.24 & $1209.8(1)$ & $2.11(15)$ & $2.9(12)$ & $51(4)$ & $73(30)$ \\
\hline & & $4^{+}$ & 309.31 & $995.67(8)$ & $4.1(2)$ & $3.97(23)$ & $100(5)$ & $100(6)$ \\
\hline & & $6^{+}$ & 628.53 & $676.4(1)^{\mathrm{b}}$ & $0.16(2)$ & & $3.9(5)$ & \\
\hline \multirow[t]{3}{*}{$0^{+}$} & 1335.9(1) & $2^{+}$ & 95.24 & $1240.8(1)$ & $3.7(2)$ & $3.84(18)$ & $100(5)$ & $100(5)$ \\
\hline & & $2^{+}$ & 952.78 & 383.1(1) & $0.47(4)$ & $0.74(8)$ & $12.7(11)$ & $19.3(21)$ \\
\hline & & $2^{+}$ & 1075.63 & $260^{\mathrm{d}}$ & $<0.10$ & $0.25(25)$ & & \\
\hline \multirow[t]{5}{*}{$\left(2^{+}, 3,4^{+}\right)$} & $1359.56(8)$ & $2^{+}$ & 95.24 & $1264.3(1)$ & $3.1(1)$ & $3.76(24)$ & $73(2)$ & $89(6)$ \\
\hline & & $4^{+}$ & 309.31 & $1050.29(9)$ & $4.25(15)$ & $4.23(14)$ & $100(3)$ & $100(3)$ \\
\hline & 1373.15(9) & $2^{+}$ & 95.24 & 1277.91(10) & $4.6(2)$ & $5.18(11)$ & $100(4)$ & $100(2)$ \\
\hline & & $2^{+}$ & 952.78 & $420.59(10)^{b}$ & $0.48(5)$ & & $10.4(11)$ & \\
\hline & $1394.3(2)^{\mathrm{e}}$ & $6^{+}$ & 628.53 & $765.8(2)^{f}$ & $0.42(4)$ & & $100(10)$ & \\
\hline \multirow[t]{2}{*}{$\left(2^{+}\right)$} & $1397.8(1)$ & $2^{+}$ & 95.24 & $1302.5(1)$ & $4.0(3)$ & $4.62(11)$ & $100(8)$ & $100(2)$ \\
\hline & & $2^{+}$ & 952.78 & $445.10(10)$ & $0.67(7)$ & $1.07(16)$ & $16.8(18)$ & $23(3)$ \\
\hline \multirow{5}{*}{$\left(4^{-}\right)$} & 1419.05(9) & $4^{+}$ & 309.31 & $1109.70(8)$ & 27.3(9) & $27.0(11)$ & $100(3)$ & $100(4)$ \\
\hline & & $6^{+}$ & 628.53 & $790^{\mathrm{d}}$ & $<0.05$ & $0.16(16)$ & & \\
\hline & & $4^{+}$ & 1129.94 & $289.13(8)$ & $3.0(1)$ & $3.07(8)$ & $11.0(4)$ & $11.4(3)$ \\
\hline & & $3^{+}$ & 1181.39 & $237.70(8)$ & $3.65(16)$ & $3.63(7)$ & $13.4(6)$ & $13.4(3)$ \\
\hline & & $4^{+}$ & 1304.97 & $114.0(1)$ & $0.39(3)$ & $0.4(4)$ & $1.4(1)$ & $1.5(15)$ \\
\hline \multirow[t]{2}{*}{$\left(5^{+}\right)$} & $1463.38(9)$ & $4^{+}$ & 309.31 & $1154.06(10)$ & $2.5(1)$ & $2.40(17)$ & $100(4)$ & $100(7)$ \\
\hline & & $6^{+}$ & 628.53 & $834.86(10)$ & $0.61(4)$ & $0.90(9)$ & $24(2)$ & $38(38)$ \\
\hline \multirow[t]{2}{*}{$\left(4^{+}, 5\right)$} & $1472.28(9)$ & $4^{+}$ & 309.31 & $1162.9(1)$ & $1.89(8)$ & $2.07(23)$ & $100(4)$ & $100(11)$ \\
\hline & & $6^{+}$ & 628.53 & $843.79(10)$ & $1.39(9)$ & $1.73(13)$ & $74(5)$ & $84(6)$ \\
\hline \multirow[t]{3}{*}{$\left(2^{+}, 3,4^{+}\right)$} & $1482.47(10)$ & $2^{+}$ & 95.24 & 1387.2(1) & $5.9(4)$ & $4.9(3)$ & $100(7)$ & $100(6)$ \\
\hline & & $4^{+}$ & 309.31 & $1173.2(1)$ & $1.3(1)$ & $1.05(12)$ & $22(2)$ & $21(2)$ \\
\hline & & $2^{+}$ & 1075.63 & $406.8(1)^{\mathrm{g}}$ & $0.25(4)$ & $0.60(5)$ & $4.2(7)$ & $12(1)$ \\
\hline \multirow[t]{6}{*}{ (2 to 5$)$} & 1496.31(10) & $4^{+}$ & 309.31 & 1186.93(9) & $5.0(2)$ & $4.89(17)$ & $100(4)$ & $100(3)$ \\
\hline & & $4^{+}$ & 1129.94 & $366.44(10)$ & $0.60(5)$ & $0.59(4)$ & $12.0(10)$ & $12.1(8)$ \\
\hline & & $3^{+}$ & 1181.39 & $314.92(10)^{\mathrm{b}}$ & $0.20(4)$ & & $4.0(8)$ & \\
\hline & $1502.9(1)^{\mathrm{h}}$ & $2^{+}$ & 95.24 & $1407.6(2)^{\mathrm{i}}$ & $1.13(15)$ & $1.36(14)$ & $72(10)$ & $72(7)$ \\
\hline & & $2^{+}$ & 1075.63 & $427.14(7)^{\mathrm{g}}$ & $1.58(16)$ & $1.9(5)$ & $100(10)$ & $100(26)$ \\
\hline & & $3^{+}$ & 1181.39 & $321.6(1)^{\mathrm{b}}$ & $0.36(5)$ & & $23(3)$ & \\
\hline \multirow[t]{2}{*}{$\left(5^{-}\right)$} & $1504.1(1)$ & $4^{+}$ & 309.31 & 1194.75(9) & $2.75(16)$ & $2.74(18)$ & $100(6)$ & $100(7)$ \\
\hline & & $6^{+}$ & 628.53 & $875.54(10)$ & $2.03(15)$ & $2.3(4)$ & $74(5)$ & $84(15)$ \\
\hline \multirow[t]{2}{*}{$\left(4^{+}\right)$} & $1535.3(1)$ & $4^{+}$ & 309.31 & $1226.05(11)$ & $4.4(3)$ & $<1.4$ & $100(7)$ & \\
\hline & & $4^{+}$ & 1129.94 & $405.3(1)^{\mathrm{b}}$ & $0.41(4)$ & & $9.3(9)$ & \\
\hline \multirow[t]{4}{*}{$\left(2^{+}, 3,4^{+}\right)$} & $1575.2(1)$ & $2^{+}$ & 95.24 & $1478.9(2)$ & $4.6(3)$ & $4.33(25)$ & $73(5)$ & $92(5)$ \\
\hline & & $4^{+}$ & 309.31 & $1266.0(1)$ & $6.3(5)$ & $4.7(3)$ & $100(8)$ & $100(6)$ \\
\hline & & $2^{+}$ & 1075.63 & $499.5(1)^{\mathrm{b}}$ & $0.37(5)$ & & $5.9(8)$ & \\
\hline & & $3^{+}$ & 1181.39 & $393.8(1)^{\mathrm{b}}$ & $0.22(3)$ & & $3.5(5)$ & \\
\hline
\end{tabular}


TABLE II. (Continued.)

\begin{tabular}{|c|c|c|c|c|c|c|c|c|}
\hline$J_{i}^{\pi}$ & $E_{i}(\mathrm{keV})$ & $J_{f}^{\pi}$ & $E_{f}(\mathrm{keV})$ & $E_{\gamma}(\mathrm{keV})$ & $I_{\gamma}$ & $I_{\mathrm{lit}}{ }^{\mathrm{a}}$ & $I_{\gamma}^{\mathrm{rel}}$ & $I_{\text {lit }}^{\mathrm{rel}}$ \\
\hline $4^{+}$ & 1601.1(1) & $4^{+}$ & 309.31 & $1291.8(2)$ & $1.08(8)$ & $1.00(11)$ & $100(7)$ & $100(11)$ \\
\hline \multirow[t]{8}{*}{$\left(3^{-}\right)$} & $1640.1(2)$ & $2^{+}$ & 95.24 & $1544.8(2)$ & 13.1(4) & $11.9(5)$ & $87(3)$ & $81(3)$ \\
\hline & & $4^{+}$ & 309.31 & $1330.7(1)$ & $15.0(5)$ & $14.7(5)$ & $100(3)$ & $100(3)$ \\
\hline & & $2^{+}$ & 952.78 & $687.4(2)^{\mathrm{b}}$ & $0.60(6)$ & & $4.0(4)$ & \\
\hline & & $2^{+}$ & 1075.63 & $564.4(1)$ & $1.25(9)$ & $1.14(7)$ & $8.3(6)$ & $7.8(5)$ \\
\hline & & $3^{+}$ & 1181.39 & $458.7(1)$ & $1.19(9)$ & $1.02(8)$ & $7.9(6)$ & $6.9(5)$ \\
\hline & & $4^{+}$ & 1304.97 & $335.2(1)$ & $0.55(4)$ & $1.26(10)$ & $3.7(3)$ & $8.6(7)$ \\
\hline & & & 1359.56 & $280.6(1)$ & $0.28(3)$ & $0.3(3)$ & $1.9(2)$ & $2(2)$ \\
\hline & & $\left(4^{-}\right)$ & 1419.05 & $221.17(7)$ & $1.66(13)$ & $2.43(10)$ & 11.1(9) & $16.5(7)$ \\
\hline \multirow[t]{30}{*}{ (2 to 5$)$} & $1684.7(1)$ & $4^{+}$ & 309.31 & $1375.4(1)$ & $3.5(2)$ & $3.78(21)$ & 100(6) & 100(6) \\
\hline & & $4^{+}$ & 1129.94 & $554.7(1)^{\mathrm{b}}$ & $0.25(4)$ & & $7.1(11)$ & \\
\hline & & $3^{+}$ & 1181.39 & $503.3(1)$ & $1.92(11)$ & $2.43(21)$ & $55(3)$ & $64(6)$ \\
\hline & & $4^{+}$ & 1304.97 & $379.8(1)$ & $0.45(6)$ & $1.62(12)$ & $12.9(2)$ & $43(3)$ \\
\hline & & & 1463.38 & $221.5(1)^{b}$ & $0.24(4)$ & & $6.9(11)$ & \\
\hline & $1691.25(10)^{\mathrm{h}}$ & $4^{+}$ & 309.31 & $1381.9(2)^{\mathrm{b}}$ & $1.0(2)$ & & $100(20)$ & \\
\hline & & $6^{+}$ & 628.53 & $1062.8(1)^{\mathrm{b}}$ & $0.30(5)$ & & $30(5)$ & \\
\hline & $1696.0(1)^{\mathrm{h}}$ & $0^{+}$ & 871.45 & $824.5(1)^{\mathrm{g}}$ & $0.53(6)$ & $0.6(6)$ & $100(11)$ & $100(100)$ \\
\hline & & $2^{+}$ & 952.78 & $742.3(1)^{\mathrm{g}}$ & $0.48(6)$ & $1.0(10)$ & 91(11) & $167(167)$ \\
\hline & & $2^{+}$ & 1075.63 & $620.4(1)^{\mathrm{g}}$ & $0.45(6)$ & $0.67(9)$ & $85(11)$ & $112(15)$ \\
\hline & $1729.1(2)^{\mathrm{h}}$ & $4^{+}$ & 309.31 & $1418.7(2)^{\mathrm{i}}$ & $1.4(2)$ & $1.0(8)$ & $96(14)$ & $67(54)$ \\
\hline & & $3^{+}$ & 1181.39 & $547.66(9)^{\mathrm{g}}$ & $1.46(8)$ & $1.50(4)$ & $100(5)$ & $100(3)$ \\
\hline & & $4^{+}$ & 1304.97 & $424.1(1)^{b}$ & $0.45(6)$ & & $31(4)$ & \\
\hline & & $\left(5^{+}\right)$ & 1463.38 & $265.7(1)^{b}$ & $0.29(4)$ & & $20(3)$ & \\
\hline & & & 1496.31 & $232.9(2)^{\mathrm{b}}$ & $0.13(4)$ & & $8.9(27)$ & \\
\hline & & $5^{-}$ & 1504.06 & $225.1(1)^{\mathrm{g}}$ & $0.19(3)$ & $0.3(3)$ & $13.0(21)$ & $20(20)$ \\
\hline & $1775.95(10)^{\mathrm{h}}$ & $4^{+}$ & 309.31 & $1466.7(1)^{\mathrm{b}}$ & $0.70(8)$ & & 109(12) & \\
\hline & & $6^{+}$ & 628.53 & $1147.4(1)^{\mathrm{i}}$ & $0.64(8)$ & $0.39(10)$ & $100(13)$ & $100(26)$ \\
\hline & $1832.35(10)^{\mathrm{h}}$ & $4^{+}$ & 309.31 & $1523.0(1)^{\mathrm{g}}$ & $0.49(6)$ & $0.61(10)$ & $100(12)$ & $100(16)$ \\
\hline & & $6^{+}$ & 628.53 & $1203.9(1)^{b}$ & $0.45(6)$ & & $92(12)$ & \\
\hline & $1902.8(1)^{\mathrm{h}}$ & $2^{+}$ & 1075.63 & $827.2(1)^{\mathrm{g}}$ & $0.45(6)$ & $0.24(24)$ & $88(12)$ & $53(53)$ \\
\hline & & $3^{+}$ & 1181.39 & $721.5(1)^{\mathrm{i}}$ & $0.24(4)$ & $1.00(12)$ & $47(8)$ & $222(27)$ \\
\hline & & $4^{+}$ & 1304.97 & $597.9(1)^{\mathrm{g}}$ & $0.51(6)$ & $0.45(10)$ & $100(12)$ & $100(22)$ \\
\hline & & $\left(5^{+}\right)$ & 1463.38 & $439.5(1)^{\mathrm{b}}$ & $0.24(4)$ & & $47(8)$ & \\
\hline & $1912.25(10)^{\mathrm{h}}$ & $2^{+}$ & 1075.63 & $836.6(1)^{b}$ & $0.84(7)$ & & $100(8)$ & \\
\hline & & $3^{+}$ & 1181.39 & $730.8(1)^{b}$ & $0.47(6)$ & & $56(7)$ & \\
\hline & & & 1482.47 & $429.8(1)^{\mathrm{i}}$ & $0.70(7)$ & $0.6(6)$ & $83(8)$ & \\
\hline & $1935.2(1)^{\mathrm{h}}$ & $2^{+}$ & 1075.63 & $859.6(1)^{b}$ & $0.38(4)$ & & $58(6)$ & \\
\hline & & $3^{+}$ & 1419.05 & $516.2(1)^{b}$ & $0.65(8)$ & & $100(12)$ & \\
\hline & & & 1482.47 & $452.7(1)^{\mathrm{b}}$ & $0.57(7)$ & & $88(11)$ & \\
\hline
\end{tabular}

\footnotetext{
${ }^{a}$ Literature values for intensities are from the evaluated ${ }^{172} \mathrm{Ta}$ EC data of Ref. [17].

${ }^{\mathrm{b}} \gamma$-ray line was not previously reported.

${ }^{\mathrm{c}}$ Spin assignment confirmed based on newly observed depopulating transition.

${ }^{\mathrm{d}} \gamma$-ray line reported [17] in this placement based only on energy difference.

${ }^{\mathrm{e}}$ Level was not previously reported in $\beta$ decay.

${ }^{\mathrm{f}} \gamma$-ray line not previously reported in $\beta$ decay.

${ }^{\mathrm{g}} \gamma$-ray line previously observed in $\beta$ decay but unplaced in level scheme.

${ }^{\mathrm{h}}$ Level was not previously reported.

${ }^{\mathrm{i}} \gamma$-ray line previously reported but in alternate placement.
}

the present work is given in Fig. 7. Spin and parity assignments are taken from Ref. [17], except where noted.

\section{1. $\left(4^{+}, 5,6^{+}\right)$level at $1031 \mathrm{keV}$}

This level was proposed [23] in $\beta$ decay on the basis of two depopulating transitions of 722 and $402 \mathrm{keV}$ to the $4_{1}^{+}$and $6_{1}^{+}$ levels, respectively. As shown in Fig. 8, a gate on the 319-keV, $6_{1}^{+} \rightarrow 4_{1}^{+}$transition finds no evidence for coincidences with a transition of $402 \mathrm{keV}$, reported with an intensity of $0.63(9)$. For comparison, the 501-keV transition highlighted in Fig. 8 does feed the $6_{1}^{+}$level with a reported intensity of 1.41(22). The 722-keV transition is found to be coincident with not only the $214-\mathrm{keV}, 4_{1}^{+} \rightarrow 2_{1}^{+}$transition, but also with an $1187-\mathrm{keV}$ 

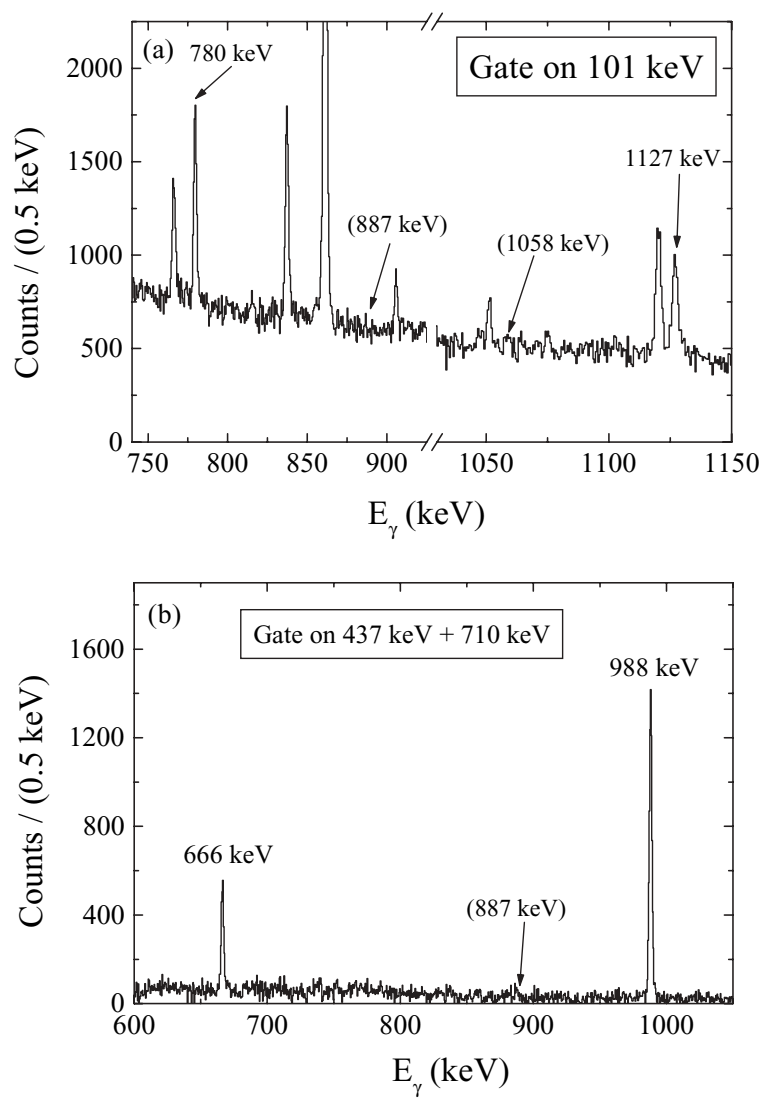

FIG. 3. (a) Spectrum gated on the $101-\mathrm{keV}, 2_{1}^{+} \rightarrow 0_{1}^{+}$transition in ${ }^{170} \mathrm{Hf}$ illustrating the unobserved coincidences with 887 - and 1058-keV transitions. (b) Composite spectrum gated on 437- and $710-\mathrm{keV}$ transitions feeding the level at $988 \mathrm{keV}$ in ${ }^{170} \mathrm{Hf}$, showing the 988-, 666-, and unobserved previously reported $887-\mathrm{keV}$ branches from the $988-\mathrm{keV}$ level.

transition (which depopulates a level at $1496 \mathrm{keV}$ and strongly feeds the $4_{1}^{+}$level), as shown in Fig. 9. Thus, the $722-\mathrm{keV}$ transition is given an alternate placement in the level scheme as directly populating the level at $1496 \mathrm{keV}$. With no evidence

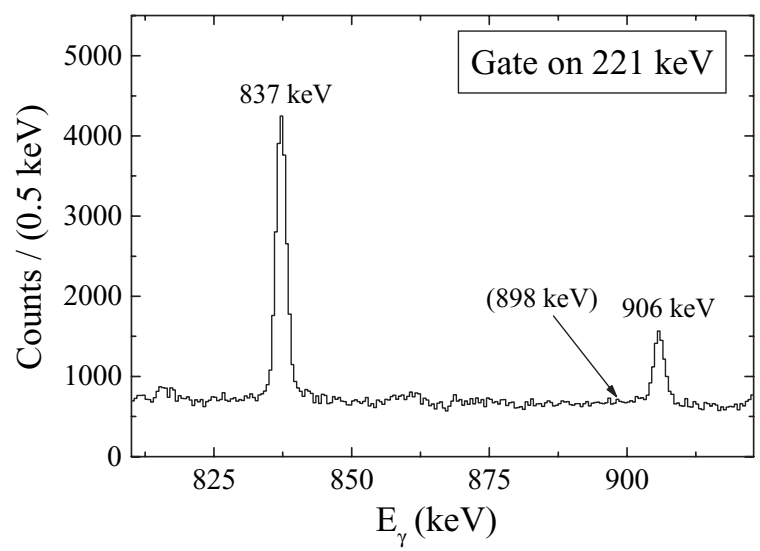

FIG. 4. Spectrum gated on the $221-\mathrm{keV}, 4_{1}^{+} \rightarrow 2_{1}^{+}$transition in ${ }^{170} \mathrm{Hf}$ illustrating the observed coincidences with $837-$ and $906-\mathrm{keV}$ transitions and the unobserved coincidence with a $898-\mathrm{keV}$ transition.

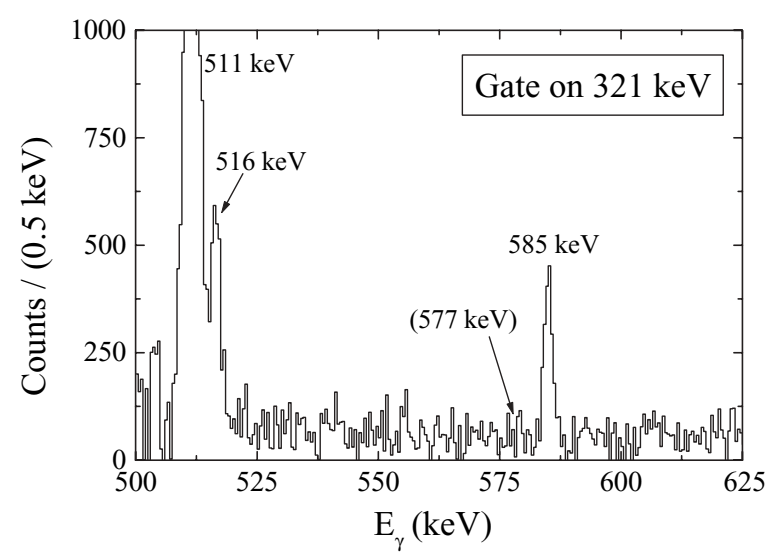

FIG. 5. Spectrum gated on the $321-\mathrm{keV}, 6_{1}^{+} \rightarrow 4_{1}^{+}$transition in ${ }^{170} \mathrm{Hf}$ illustrating the observed coincidences with 516- and 585-keV transitions and the unobserved coincidence with a 577-keV transition.

for any of the proposed depopulating transitions, we eliminate the level at $1031 \mathrm{keV}$.

\section{2. $\left(2^{+}\right)$level at $1076 \mathrm{keV}$}

The level at $1076 \mathrm{keV}$ was previously reported [17] to decay by two transitions of 980 and $1076 \mathrm{keV}$, to the $2_{1}^{+}$and $0_{1}^{+}$levels, respectively, and assigned as the bandhead of the
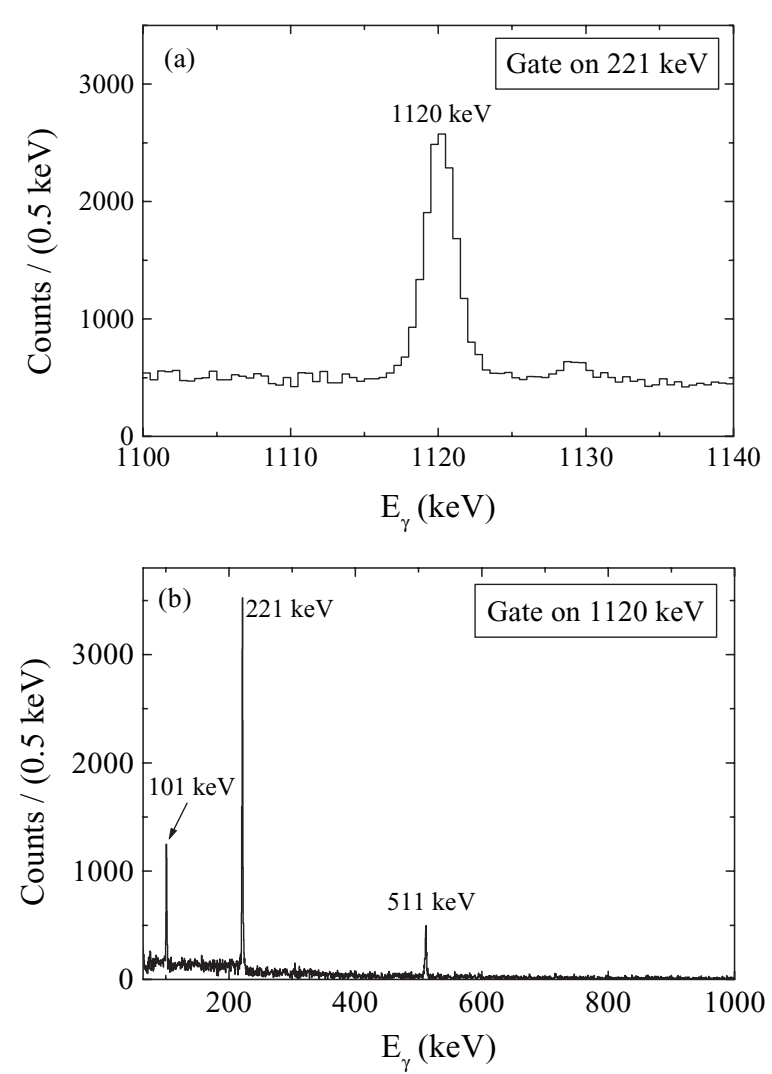

FIG. 6. Spectra providing evidence for the new placement of a $1120-\mathrm{keV}$ transition in ${ }^{170} \mathrm{Hf}$. (a) Spectrum gated on the $221-\mathrm{keV}$, $4_{1}^{+} \rightarrow 2_{1}^{+}$transition. (b) Spectrum gated on the $1120-\mathrm{keV}$ transition. 


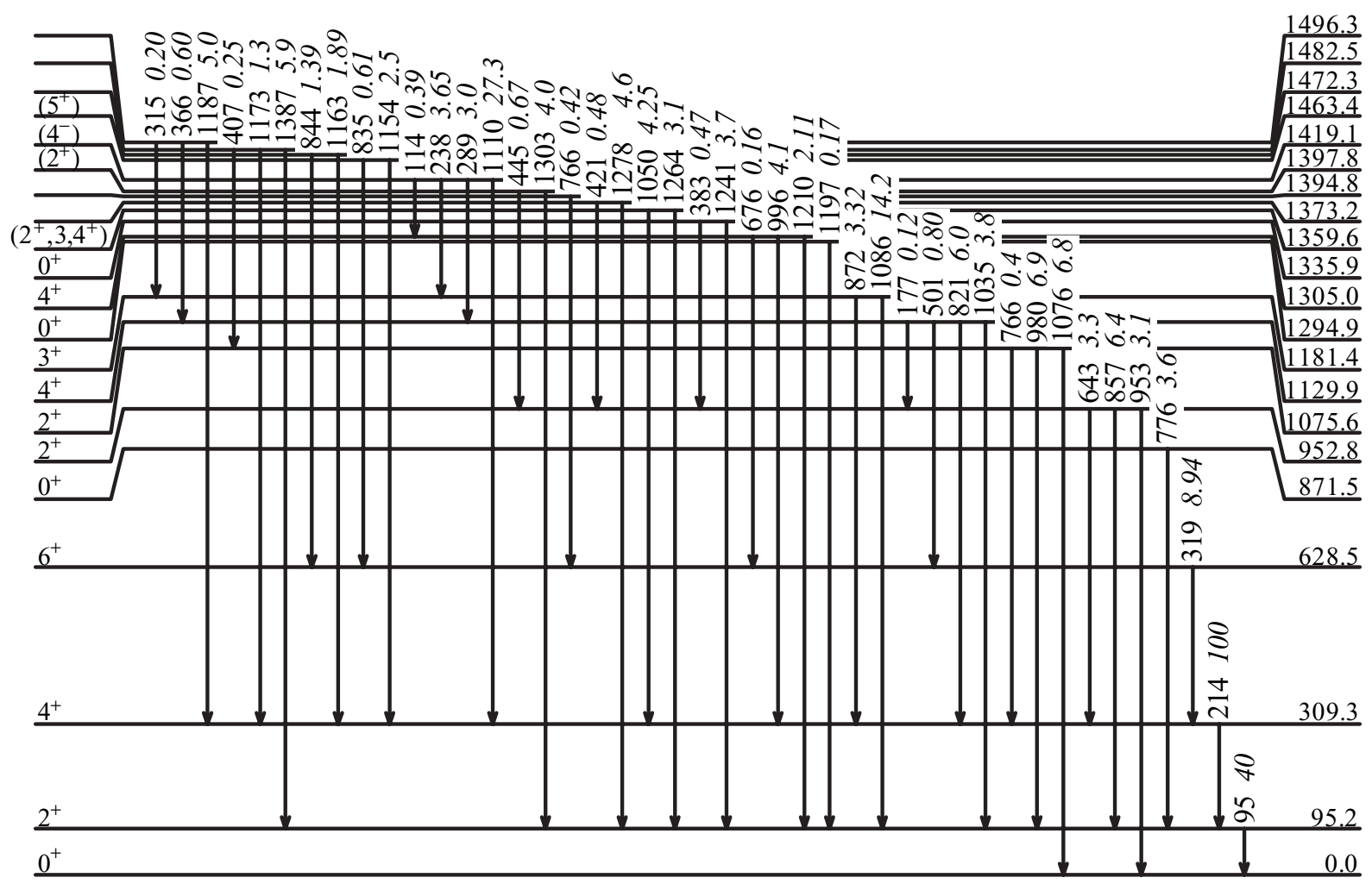

${ }^{172} \mathrm{Hf}$

FIG. 7. Level scheme for ${ }^{172} \mathrm{Hf}$ populated in the $\beta^{+} / \epsilon$ decay of ${ }^{172} \mathrm{Ta}$ for levels below $1500 \mathrm{keV}$. Levels are marked with their energy in keV. $\gamma$-ray transitions are labeled by their energy in $\mathrm{keV}$ and relative intensities (in italics), normalized to the $214-\mathrm{keV}, 4_{1}^{+} \rightarrow 22_{1}^{+}$transition $\left(I_{214} \equiv 100\right)$.

$\gamma$ band. These two transitions are confirmed and found to have intensities consistent with those given in the literature. An additional transition from this level to the $4_{1}^{+}$state is also observed. A summed gate on the 427- and 564-keV transitions that feed the level at $1076 \mathrm{keV}$ is given in Fig. 10. Strong coincidences are observed with the 980- and 1076-keV transitions, as well as a weak coincidence with a $766-\mathrm{keV}$ transition. A 766.2(1)-keV line with intensity $0.40(1)$ is now placed as a transition from the level at $1076 \mathrm{keV}$ to the $4_{1}^{+}$level. The observation of the transition to the $4_{1}^{+}$state confirms the previous spin assignment of $2^{+}$for this level. We note that the $766-\mathrm{keV}$ line was established as a doublet in the present work, as also depopulating a level at $1394 \mathrm{keV}$. This level is discussed separately below.

\section{3. $4^{+}$level at $1130 \mathrm{keV}$}

This level was previously observed [23] to decay to the $2_{1}^{+}, 4_{1}^{+}$, and $6_{1}^{+}$levels by transitions of 1035,821 , and $501 \mathrm{keV}$, respectively, and assigned as the $4^{+}$member of the $0_{2}^{+}$ band. The previously reported depopulating transitions were observed and found to have intensities consistent with the literature. An additional transition of $177 \mathrm{keV}$ to the $2_{2}^{+}$state was also observed, corresponding to an intraband transition within the excited $0^{+}$band. Evidence for this transition is given in Fig. 11. In a summed gate on the 289- and 366-keV transitions [Fig. 11(a)] populating the level at $1130 \mathrm{keV}$, the strong 1035-, 821-, and 501-keV depopulating transitions are observed, along with the weaker $177-\mathrm{keV}$ transition. In addition, a weak $177-\mathrm{keV}$ transition is seen in coincidence with the 857-keV, $2_{2}^{+} \rightarrow 2_{1}^{+}$transition, as shown in Fig. 11(b).

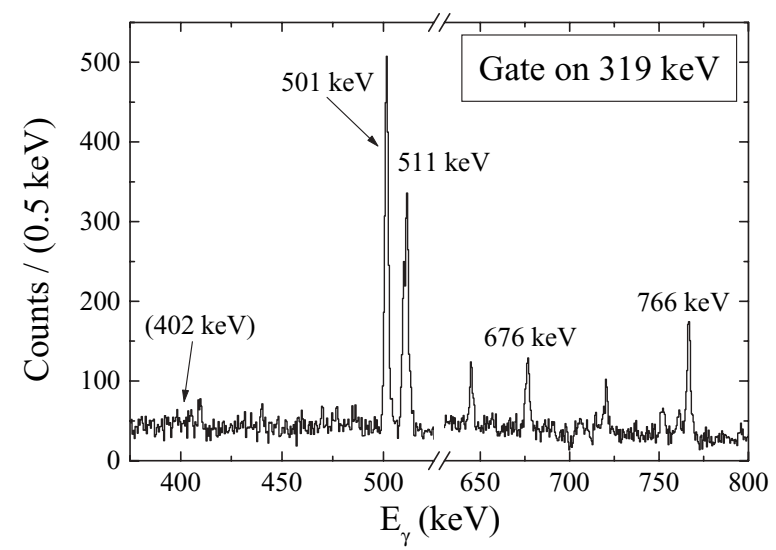

FIG. 8. Spectrum gated on the $319-\mathrm{keV}, 6_{1}^{+} \rightarrow 4_{1}^{+}$transition in ${ }^{172} \mathrm{Hf}$, illustrating observed coincidences with 501-, 676-, and 766-keV transitions and the unobserved coincidence with a 402-keV transition. 


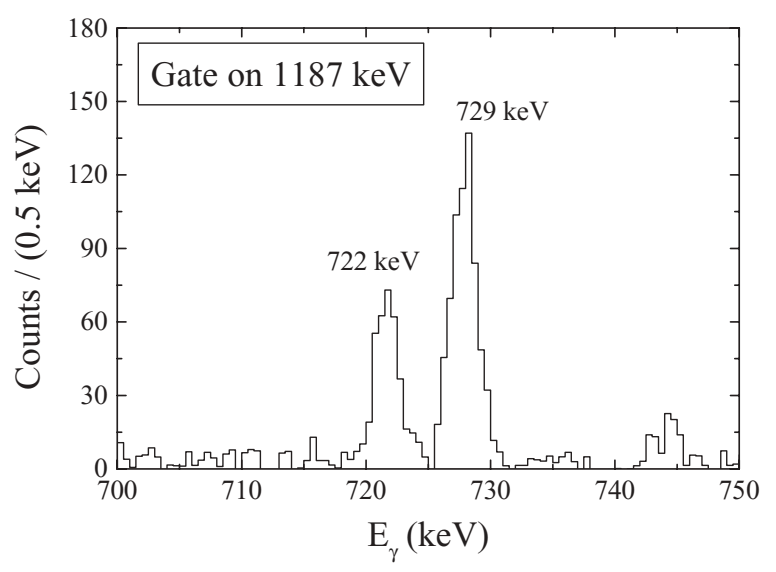

FIG. 9. Spectrum providing evidence for the new placement of a 722-keV transition in ${ }^{172} \mathrm{Hf}$. Spectrum is obtained by gating on the $1187-\mathrm{keV}$ transition in ${ }^{172} \mathrm{Hf}$, illustrating strong coincidences with a 722-keV transition.

\section{4. $\left(4^{+}\right)$level at $1305 \mathrm{keV}$}

The level at $1305 \mathrm{keV}$ was previously proposed [23] to decay by transitions of 995 and $1209 \mathrm{keV}$ to the $4_{1}^{+}$and $2_{1}^{+}$levels, respectively, and assigned as the $4^{+}$member of the $\gamma$ band. These depopulating transitions are confirmed in the present experiment. An additional $676-\mathrm{keV}$ depopulating transition is observed to the $6_{1}^{+}$level. A $676-\mathrm{keV}$ transition is found to be in coincidence with the $319-\mathrm{keV}, 6_{1}^{+} \rightarrow 4_{1}^{+}$ transition, as shown in Fig. 8. The observation of a new transition decaying to the $6_{1}^{+}$level confirms the previous spin assignment of $4^{+}$to this level.

\section{5. $0^{+}$level at $1335 \mathrm{keV}$}

This level was identified in $\beta$ decay [23] on the basis of two depopulating transitions of 1241 and $383 \mathrm{keV}$ to the $2_{1}^{+}$and $2_{2}^{+}$levels, respectively. These transitions were confirmed in the present work. The evaluated data [17] added a previously unplaced transition [23] of $260 \mathrm{keV}$ to the $2_{3}^{+}$level based only

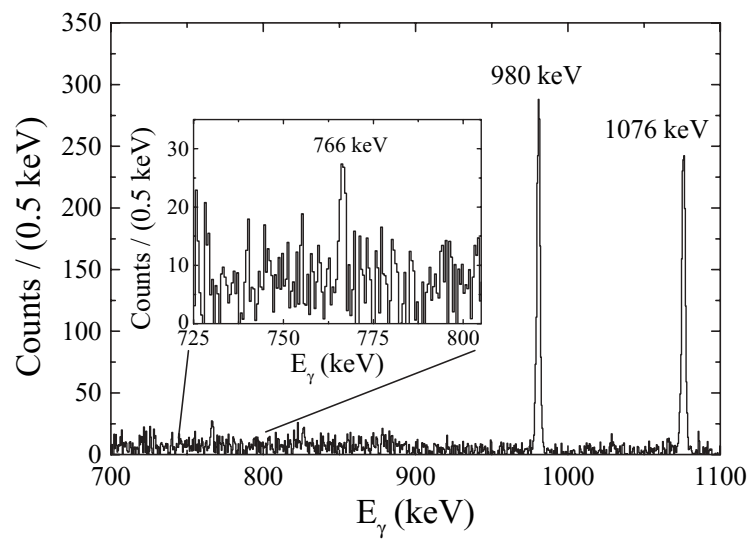

FIG. 10. Spectrum gated on the 427- and 564-keV transitions that populate the level at $1075 \mathrm{keV}$ in ${ }^{172} \mathrm{Hf}$. Coincidences are observed with the previously reported depopulating transitions of 980 and $1076 \mathrm{keV}$, as well a newly observed 766-keV depopulating transition. on the energy difference. The present coincidence data find no evidence for a $260-\mathrm{keV}$ transition from this level to the level at $1076 \mathrm{keV}$.

\section{Level at $1373 \mathrm{keV}$}

A level at $1373 \mathrm{keV}$ was previously tentatively proposed [17] on the basis of a single depopulating transition of $1277 \mathrm{keV}$ to the $2_{1}^{+}$state. The placement and intensity of this transition were confirmed in the present experiment. The existence of this level is further supported by the observation of an additional depopulating transition of $421 \mathrm{keV}$ to the 953-keV level.

\section{Level at $1394 \mathrm{keV}$}

A level at $1394 \mathrm{keV}$ was previously observed only in the $(\alpha, \mathrm{x} n)$ reaction [17] with a single depopulating transition of $766 \mathrm{keV}$ to the $6_{1}^{+}$state. In the present work, we also observed a 766-keV transition in coincidence with the $319-\mathrm{keV}, 6_{1}^{+} \rightarrow 4_{1}^{+}$ transition, as shown in Fig. 8. Thus, we place a 765.8(2)-keV transition with intensity $0.42(4)$ depopulating a level at $1394 \mathrm{keV}$, which is mostly likely associated with the previously observed level at $1394 \mathrm{keV}$ in the $(\alpha, \mathrm{x} n)$ reaction.

\section{Levels in ${ }^{174} \mathrm{Hf}$}

${ }^{174} \mathrm{Hf}$ has been studied previously in significant detail in four different $\beta$-decay experiments [24-27]. The present experiment finds mostly agreement with the literature data on ${ }^{174} \mathrm{Hf}$. The level scheme of ${ }^{174} \mathrm{Hf}$ populated in $\beta$ decay extends up to $3.2 \mathrm{MeV}$ with many of the levels above $2 \mathrm{MeV}$ decaying by high-energy $\gamma$ rays $\left(E_{\gamma}>2 \mathrm{MeV}\right)$. In the present experiment, the $\gamma$-ray detection energy range was limited to a maximum of $2 \mathrm{MeV}$ and thus we have no ability to confirm a large portion of the level scheme. For that reason, we limit the discussion to levels below $1500 \mathrm{keV}$, which includes all the relevant levels for investigating the low-spin members of the $\gamma$ and $\beta$ bands. Table III lists the levels below $1500 \mathrm{keV}$ populated in ${ }^{174} \mathrm{Hf}$ and their $\gamma$ decay. Following the convention of Ref. [18], intensities are normalized to the intensity of the 206-keV, $4_{1}^{+} \rightarrow 2_{1}^{+}$transition $\left(I_{206} \equiv 1000\right)$. The level scheme for ${ }^{174} \mathrm{Hf}$ for levels below $1500 \mathrm{keV}$ deduced in the present work is given in Fig. 12. Spin and parity assignments are taken from Ref. [18].

\section{1. $4^{+}$level at $1062 \mathrm{keV}$}

The level at $1062 \mathrm{keV}$ was previously reported [18] to decay by three transitions of 971,765 , and $454 \mathrm{keV}$ to the $2_{1}^{+}, 4_{1}^{+}$, and $6_{1}^{+}$levels, respectively. These transitions were observed and confirmed to have intensities consistent with those from the literature. An additional depopulating transition of $162-\mathrm{keV}$ to the $2_{2}^{+}$state at $900 \mathrm{keV}$ was also observed in the present experiment. Support for this transition is given in Fig. 13. A 162-keV transition is found in coincidence with the $809-\mathrm{keV}, 2_{2}^{+} \rightarrow 2_{1}^{+}$transition, which depopulates the level 
TABLE III. Levels populated in ${ }^{174} \mathrm{Hf}$ and their $\gamma$ decay. Relative intensities (in $\beta$ decay) are given for $\gamma$-ray transitions depopulating the levels and compared with literature values [18] where available.

\begin{tabular}{|c|c|c|c|c|c|c|c|c|}
\hline$J_{i}^{\pi}$ & $E_{i}(\mathrm{keV})$ & $J_{f}^{\pi}$ & $E_{f}(\mathrm{keV})$ & $E_{\gamma}(\mathrm{keV})$ & $I_{\gamma}$ & $I_{\text {lit }}{ }^{\mathrm{a}}$ & $I_{\gamma}^{\mathrm{rel}}$ & $I_{\text {lit }}^{\mathrm{rel}}$ \\
\hline $2^{+}$ & $91.02(5)$ & $0^{+}$ & 0.00 & $91.02(5)$ & $300(30)$ & $262(20)$ & $100(2)$ & $100(3)$ \\
\hline $4^{+}$ & $297.50(6)$ & $2^{+}$ & 91.02 & $206.48(4)$ & $1000(20)$ & $1000(20)$ & $100(2)$ & $100(3)$ \\
\hline $6^{+}$ & $608.32(6)$ & $4^{+}$ & 297.50 & $310.82(6)$ & $20.3(8)$ & $20.8(8)$ & $100(4)$ & $100(4)$ \\
\hline $0^{+}$ & $828.32(7)$ & $2^{+}$ & 91.02 & 737.3(1) & $0.95(25)$ & $0.86(23)$ & $100(25)$ & $100(27)$ \\
\hline \multirow[t]{3}{*}{$2^{+}$} & $900.47(7)$ & $0^{+}$ & 0.00 & $900.48(8)$ & $8.5(5)$ & $8.9(6)$ & $68(4)$ & $73(5)$ \\
\hline & & $2^{+}$ & 91.02 & $809.48(7)$ & $12.5(7)$ & $12.2(7)$ & $100(6)$ & $100(6)$ \\
\hline & & $4^{+}$ & 297.50 & $602.92(8)$ & $7.6(6)$ & $7.2(6)$ & $61(5)$ & $59(5)$ \\
\hline \multirow[t]{4}{*}{$4^{+}$} & $1062.36(7)$ & $2^{+}$ & 91.02 & $971.31(8)$ & $19.3(10)$ & $18.2(11)$ & $87(5)$ & $87(5)$ \\
\hline & & $4^{+}$ & 297.50 & $764.89(8)$ & $22.2(10)$ & $21.0(10)$ & $100(5)$ & $100(5)$ \\
\hline & & $6^{+}$ & 608.32 & $454.05(9)$ & $4.2(4)$ & $3.6(5)$ & $19(2)$ & $17(2)$ \\
\hline & & $2^{+}$ & 900.47 & $162.0(1)^{\mathrm{b}}$ & $0.47(7)$ & & $2.1(3)$ & \\
\hline \multirow[t]{3}{*}{$2^{+}$} & $1226.94(8)$ & $0^{+}$ & 0.00 & $1226.90(8)$ & $9.8(6)$ & $11(5)$ & $86(5)$ & $97(44)$ \\
\hline & & $2^{+}$ & 91.02 & $1135.96(8)$ & $11.4(7)$ & 11.3(9) & $100(6)$ & $100(8)$ \\
\hline & & $4^{+}$ & 297.50 & $928.9(1)^{b}$ & $0.40(8)$ & & $3.5(7)$ & \\
\hline \multirow[t]{2}{*}{$\left(3^{+}\right)$} & $1303.78(8)$ & $2^{+}$ & 91.02 & $1212.74(9)$ & $13.0(10)$ & $10.7(13)$ & $100(8)$ & $100(12)$ \\
\hline & & $4^{+}$ & 297.50 & $1006.3(1)$ & $2.3(2)$ & $1.2(5)$ & $18(2)$ & $11(5)$ \\
\hline \multirow[t]{2}{*}{$\left(2^{-}\right)$} & 1308.90(9) & $2^{+}$ & 91.02 & $1217.87(8)$ & $9.1(8)$ & 7.2(9) & $100(9)$ & $100(13)$ \\
\hline & & $2^{+}$ & 900.47 & 408.43(8) & $0.45(10)$ & $0.79(25)$ & $4.9(11)$ & $11.0(35)$ \\
\hline \multirow[t]{4}{*}{$\left(2^{+}\right)$} & 1319.65(9) & $0^{+}$ & 0.00 & $1319.7(2)$ & $2.4(3)$ & $1.8(3)$ & $8.2(10)$ & $7.5(13)$ \\
\hline & & $2^{+}$ & 91.02 & $1228.63(8)$ & $29.4(26)$ & $24(6)$ & $100(9)$ & $100(25)$ \\
\hline & & $4^{+}$ & 297.50 & $1022.20(8)$ & 12.1(10) & $13.8(12)$ & $41(3)$ & $58(5)$ \\
\hline & & $0^{+}$ & 828.32 & 491.3(1) & $0.28(9)$ & $0.67(19)$ & $0.95(30)$ & $2.8(8)$ \\
\hline \multirow[t]{2}{*}{$\left(3^{+}\right)$} & $1336.62(10)$ & $2^{+}$ & 91.02 & $1245.56(9)$ & $10.8(9)$ & 11(1) & $100(8)$ & $100(9)$ \\
\hline & & $4^{+}$ & 297.50 & $1039.15(9)$ & $2.3(3)$ & $2.7(4)$ & $21(3)$ & $25(4)$ \\
\hline \multirow[t]{2}{*}{$\left(4^{+}\right)$} & 1394.93(10) & $2^{+}$ & 91.02 & $1303.9(1)$ & $4.9(5)$ & $4.9(16)$ & $82(8)$ & $71(23)$ \\
\hline & & $4^{+}$ & 297.50 & 1097.43(9) & $6.0(5)$ & $6.9(5)$ & $100(8)$ & $100(7)$ \\
\hline \multirow[t]{2}{*}{$\left(4^{-}\right)$} & $1425.78(10)$ & $4^{+}$ & 297.50 & $1128.25(10)$ & $8.4(8)$ & 10.1(9) & $100(10)$ & 100(9) \\
\hline & & $4^{+}$ & 1062.36 & 363.45 & $0.75(9)$ & $0.8(2)$ & $8.9(11)$ & $7.9(20)$ \\
\hline \multirow[t]{2}{*}{$\left(5^{-}\right)$} & $1443.0(1)$ & $4^{+}$ & 297.50 & $1145.5(1)$ & $4.3(5)$ & $4.6(6)$ & $81(9)$ & $140(18)$ \\
\hline & & $6^{+}$ & 608.32 & $834.64(8)$ & $5.3(5)$ & $3.3(6)$ & $100(9)$ & $100(18)$ \\
\hline \multirow[t]{3}{*}{$\left(4^{+}\right)$} & 1449.07(9) & $2^{+}$ & 91.02 & $1358.0(1)$ & $15.0(15)$ & $14.3(20)$ & $83(8)$ & $78(11)$ \\
\hline & & $4^{+}$ & 297.50 & $1151.56(9)$ & 18.1(9) & $18.3(21)$ & $100(5)$ & $100(11)$ \\
\hline & & $6^{+}$ & 608.32 & $840.8(1)$ & $0.80(15)$ & $1.3(5)$ & $4.4(8)$ & $7.1(27)$ \\
\hline \multirow[t]{4}{*}{$\left(2^{+}\right)$} & $1496.3(2)$ & $0^{+}$ & 0.00 & $1496.4(2)$ & $1.0(4)$ & $1.4(5)$ & $45(18)$ & $47(17)$ \\
\hline & & $2^{+}$ & 91.02 & $1405.2(2)$ & $0.75(25)$ & $0.93(30)$ & $34(11)$ & $31(10)$ \\
\hline & & $4^{+}$ & 297.50 & 1198.9(1) & $2.2(5)$ & $3.0(6)$ & $100(23)$ & $100(20)$ \\
\hline & & $2^{+}$ & 900.47 & $595.8(1)$ & $0.63(10)$ & $2.54(30)^{\mathrm{c}}$ & $29(5)$ & $85(10)$ \\
\hline \multirow[t]{4}{*}{$\left(4^{+}\right)$} & $1503.44(8)$ & $2^{+}$ & 91.02 & $1412.6(2)$ & $2.0(4)$ & $3.6(6)$ & $3.0(6)$ & $4.4(7)$ \\
\hline & & $4^{+}$ & 297.50 & $1205.94(8)$ & $66(4)$ & $81(4)$ & $100(6)$ & $100(5)$ \\
\hline & & $6^{+}$ & 608.32 & $898.15(10)^{\mathrm{b}}$ & $0.64(8)$ & & $0.97(12)$ & \\
\hline & & $4^{+}$ & 1062.36 & $441.04(8)$ & $3.2(3)$ & $3.5(4)$ & $4.8(5)$ & $4.3(4)$ \\
\hline
\end{tabular}

${ }^{\mathrm{a}}$ Literature values for intensities are from the evaluated ${ }^{174} \mathrm{Ta}$ EC data of Ref. [18].

${ }^{\mathrm{b}} \gamma$-ray line was not previously reported.

${ }^{c} \gamma$-ray line previously multiply placed, undivided intensity given.

at $900 \mathrm{keV}$, as shown in Fig. 13(a). A gate on the 441-keV transition that strongly feeds the level at $1062 \mathrm{keV}$, as shown in Fig. 13(b), finds coincidences with the previously reported 971-, 765-, and 454-keV depopulating transitions, as well as a weak coincidence with a $162-\mathrm{keV}$ transition. The $900-$ and 1062-keV levels have been assigned [18] as the $2^{+}$and $4^{+}$members of the excited $K=0^{+}$band, thus the $162-\mathrm{keV}$ transition represents the intraband transition.

\section{2. $2^{+}$level at $1227 \mathrm{keV}$}

The level at $1227 \mathrm{keV}$ was reported [18] in $\beta$ decay with two depopulating transitions of 1136 and $1227 \mathrm{keV}$ to the $2_{1}^{+}$and $0_{1}^{+}$levels, respectively. These transitions were confirmed in the present work. The $1227-\mathrm{keV}$ transition is a known doublet and previously reported [18] with a significant error. From the new coincidence data, we were able to determine the intensity of the $1227-\mathrm{keV}$ transition with a significantly reduced error. 

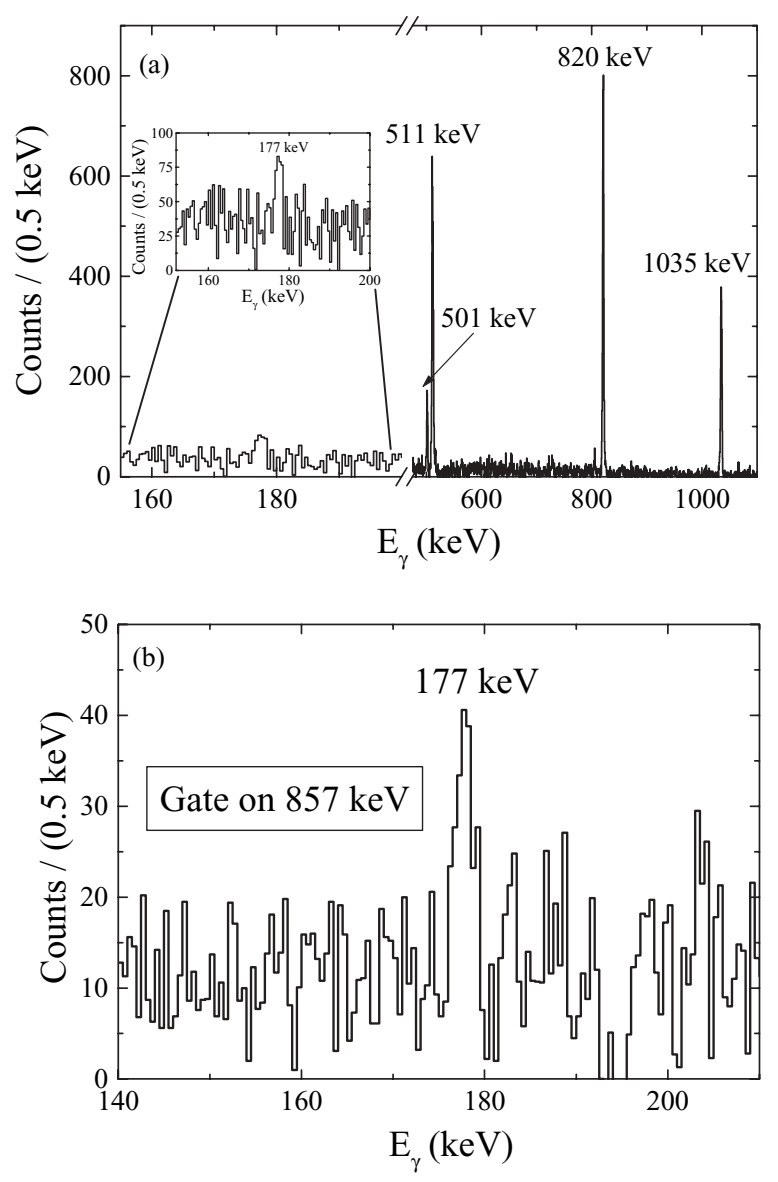

FIG. 11. Spectra providing evidence for the placement of a newly observed $177-\mathrm{keV}$ transition in ${ }^{172} \mathrm{Hf}$. (a) Summed spectrum gated on the 289- and 366-keV transitions that populate the level at $1130 \mathrm{keV}$. (b) Spectra gated on the 857-keV, $2_{2}^{+} \rightarrow 2_{1}^{+}$transition.

An additional depopulating transition of $929 \mathrm{keV}$ to the $4_{1}^{+}$ state was also observed in the present work. Evidence for this transition is given in Fig. 14 where a gate is placed on the strong $1176-\mathrm{keV}$ transition that populates the $1227-\mathrm{keV}$ level. Strong coincidences are observed with all three transitions depopulating the level at $1227 \mathrm{keV}$, including the previously unreported 929-keV transition.

\section{DISCUSSION}

In following, we examine the extent to which the Hf isotopic chain can be explained in terms of two-level mixing between $\beta$ and $\gamma$ vibrational $2^{+}$excitations. This first requires assignments of the observed levels to members of the $\beta$ and $\gamma$ excitations. These assignments are taken from the literature [14,15] and compiled data [16-18]. We start by considering the branching ratios from the excited $2^{+}$states, because electromagnetic transition strengths provide the most stringent test of structure. Plotted in Fig. 15 are the branching ratios $B\left(E 2 ; 2_{i}^{+} \rightarrow 0_{1}^{+}\right) / B\left(E 2 ; 2_{i}^{+} \rightarrow 4_{1}^{+}\right)$for ${ }^{166} \mathrm{Hf}$ through ${ }^{174} \mathrm{Hf}$. This branching ratio was chosen because it involves pure E2 transitions, so knowledge of the multipole mixing ratio is not necessary and also because the Alaga predictions are quite different: 14.3 for decay from the $2_{\gamma}^{+}$level and 0.39 for decay from the $2_{\beta}^{+}$level.

The values shown in Fig. 15 confirm the assumption of two-level mixing between the $2_{\beta}^{+}$and $2_{\gamma}^{+}$levels. In ${ }^{166} \mathrm{Hf}$ and ${ }^{168} \mathrm{Hf}$, the $2_{3}^{+}$levels have values for the branching ratio similar to the $2_{\beta}^{+}$Alaga limit, whereas the $2_{2}^{+}$branching ratios (although only lower limits) are significantly larger, suggesting they correspond to the $2_{\gamma}^{+}$state. At ${ }^{170} \mathrm{Hf}$, where the energies of the $2_{\beta}^{+}$and $2_{\gamma}^{+}$levels are nearly equal, enhanced mixing of these states is expected and both branching ratios are rather small. Above ${ }^{170} \mathrm{Hf}$, the character of the excited $2^{+}$states reverses, with $2_{3}^{+}$becoming the $2_{\gamma}^{+}$excitation (large values for the branching ratio) and the $2_{2}^{+}$resembling the decay of the $\beta$ excitation (small values for the branching ratio).

Beyond the qualitative analysis given in Fig. 15, we also consider a more rigorous treatment through the explicit bandmixing formalism [3]. Incorporating both $\gamma$-ground and $\gamma-\beta$ mixing, the $B(E 2)$ branching ratios from the $\gamma$ band to the ground band are given by [3]

$$
\begin{aligned}
& \frac{B\left(E 2 ; 2_{\gamma}^{+} \rightarrow 0_{1}^{+}\right)}{B\left(E 2 ; 2_{\gamma}^{+} \rightarrow 4_{1}^{+}\right)}=14.3 \frac{\left[1-Z_{\gamma}+2 Z_{\beta \gamma}\right]^{2}}{\left[1+9 Z_{\gamma}+12 Z_{\beta \gamma}\right]^{2}} \\
& \frac{B\left(E 2 ; 2_{\gamma}^{+} \rightarrow 2_{1}^{+}\right)}{B\left(E 2 ; 2_{\gamma}^{+} \rightarrow 0_{1}^{+}\right)}=1.43 \frac{\left[1+Z_{\gamma}-2 Z_{\beta \gamma}\right]^{2}}{\left[1-Z_{\gamma}+2 Z_{\beta \gamma}\right]^{2}},
\end{aligned}
$$

where $Z_{\gamma}$ is a measure of the mixing between the ground and $\gamma$ bands and $Z_{\beta \gamma}$ is a measure of the mixing between the $\gamma$ and $\beta$ bands. The correction factors for the $B\left(E 2 ; 2_{\gamma}^{+} \rightarrow\right.$ $\left.2_{1}^{+}\right) / B\left(E 2 ; 2_{\gamma}^{+} \rightarrow 4_{1}^{+}\right)$branching ratio can be obtained from the ratio of Eqs. (1) and (2).

Because the odd spin members of the $\gamma$ band do not mix with the $\beta$ band, the branching ratio from the $3^{+}$of the $\gamma$ band depends only on the mixing parameter $Z_{\gamma}$ given by [3]

$$
\frac{B\left(E 2 ; 3_{\gamma}^{+} \rightarrow 2_{1}^{+}\right)}{B\left(E 2 ; 3_{\gamma}^{+} \rightarrow 4_{1}^{+}\right)}=2.5 \frac{\left[1-Z_{\gamma}\right]^{2}}{\left[1+6 Z_{\gamma}\right]^{2}} .
$$

Equation (3) is first used to extract a value for $Z_{\gamma}$. Then with the extracted $Z_{\gamma}$ value, Eqs. (1) and (2) are used to determine a value of $Z_{\beta \gamma}$. The values of $Z_{\gamma}$ and $Z_{\beta \gamma}$ are summarized in Table IV for ${ }^{168} \mathrm{Hf}$ through ${ }^{174} \mathrm{Hf}$. For determining $Z_{\beta \gamma}$, all possible branching ratios from the $2_{\gamma}^{+}$level were considered.

In the case of ${ }^{168} \mathrm{Hf}$, the $2_{\gamma}^{+} \rightarrow 4_{1}^{+}$transition is not observed and reported with only an upper limit. The upper limit is used in the second column of Table IV, giving inconsistent values of $Z_{\beta \gamma}$. If, however, the upper limit is reduced by a factor of 4 (effectively multiplying the first and last branching ratios by a factor of 4 ) all the $Z_{\beta \gamma}$ values converge to a similar value. For both ${ }^{172,174} \mathrm{Hf}$, the $E 2 / M 1$ mixing ratio has not been measured for the $2_{\gamma}^{+} \rightarrow 2_{1}^{+}$transition. The values in the column labeled "Pure E2" assume pure E2 character for the $2_{\gamma}^{+} \rightarrow 2_{1}^{+}$transition. In the neighboring column, the intensity of the $2_{\gamma}^{+} \rightarrow 2_{1}^{+}$transition is reduced by the amount indicated in Table IV. Reducing the E2 component by 0.60 and 0.80 for ${ }^{172} \mathrm{Hf}$ and ${ }^{174} \mathrm{Hf}$, respectively, results in $Z_{\beta \gamma}$ values that converge for all measured transitions. Note that this procedure 
TABLE IV. $Z_{\beta \gamma}$ values extracted from branching ratios from the $2_{\gamma}^{+}$levels in ${ }^{168-174} \mathrm{Hf}$. The column labeled Exp gives the experimental branching ratio used in the calculation of $Z_{\beta \gamma}$. Errors are omitted for some $Z_{\beta \gamma}$ values, where the intensity of one of the transitions in the branching ratio is not precisely known. The $Z_{\gamma}$ values, determined from Eq. (3), are also given for each isotope.

\begin{tabular}{|c|c|c|c|c|c|c|c|c|c|c|c|}
\hline \multirow[t]{2}{*}{ Ratio } & \multicolumn{3}{|c|}{$\begin{array}{c}{ }^{168} \mathrm{Hf} \\
Z_{\gamma}=0.057(12)\end{array}$} & \multicolumn{2}{|c|}{$\begin{array}{c}{ }^{170} \mathrm{Hf} \\
Z_{\gamma}=0.084(7)\end{array}$} & \multicolumn{3}{|c|}{$\begin{array}{c}{ }^{172} \mathrm{Hf} \\
Z_{\gamma}=0.044(5)\end{array}$} & \multicolumn{3}{|c|}{$\begin{array}{c}{ }^{174} \mathrm{Hf} \\
Z_{\gamma}=0.027(10)\end{array}$} \\
\hline & Exp & $\begin{array}{c}Z_{\beta \gamma} \\
E 2\end{array}$ & $\begin{array}{c}Z_{\beta \gamma} \\
4 \times \text { Limit }\end{array}$ & Exp & $\begin{array}{l}Z_{\beta \gamma} \\
E 2\end{array}$ & Exp & $\begin{array}{l}Z_{\beta \gamma} \\
E 2\end{array}$ & $\begin{array}{c}Z_{\beta \gamma} \\
0.6 \times E 2\end{array}$ & Exp & $\begin{array}{c}Z_{\beta \gamma} \\
E 2\end{array}$ & $\begin{array}{c}Z_{\beta \gamma} \\
0.8 \times E 2\end{array}$ \\
\hline$\frac{B\left(E 2 ; 2_{\gamma}^{+} \rightarrow 0_{1}^{+}\right)}{B\left(E 2 ; 2_{\gamma}^{+} \rightarrow 4_{1}^{+}\right)}$ & $>7$ & -0.018 & -0.079 & $0.071(8)$ & -0.37 & $3.1(8)$ & $+0.09(3)$ & $+0.09(3)$ & $6.1(9)$ & $+0.035(13)$ & $+0.035(13)$ \\
\hline$\frac{B\left(E 2 ; 2_{\gamma}^{+} \rightarrow 2_{1}^{+}\right)}{B\left(E 2 ; 2_{\gamma}^{+} \rightarrow 4_{1}^{+}\right)}$ & $>27$ & -0.040 & -0.082 & 2.3 & +0.41 & 5.0 & +0.050 & +0.084 & 9.7 & +0.019 & +0.031 \\
\hline
\end{tabular}

is unique in that varying the $E 2$ strength in the above transitions by different percentages does not result in a convergence of all $Z_{\beta \gamma}$ values.

The results given in Table IV show a systematic variation of the $Z_{\beta \gamma}$ values across the Hf isotopes. Where the $\gamma$ band is below the $\beta$ band, as in ${ }^{168} \mathrm{Hf}, Z_{\beta \gamma}$ is negative and rather large. In ${ }^{170} \mathrm{Hf}$, where $2_{\beta}^{+}$and $2_{\gamma}^{+}$are nearly degenerate, the $Z_{\beta \gamma}$ values clearly indicate considerable mixing. The fact that they are so large also suggests that simple first-order perturbation analysis does not apply in this case. In ${ }^{172} \mathrm{Hf}$ and ${ }^{174} \mathrm{Hf}$, where the $\gamma$ band rises above the $\beta$ band, the $Z_{\beta \gamma}$ values are positive and show the trend of decreasing in magnitude as the energy separation between the $2_{\gamma}^{+}$and $2_{\beta}^{+}$states increases.

From these $Z_{\beta \gamma}$ values, a value for the absolute mixing matrix element between the $2_{\beta}^{+}$and $2_{\gamma}^{+}$excitations can be determined. The mixing amplitude of the unperturbed $\beta$ in the

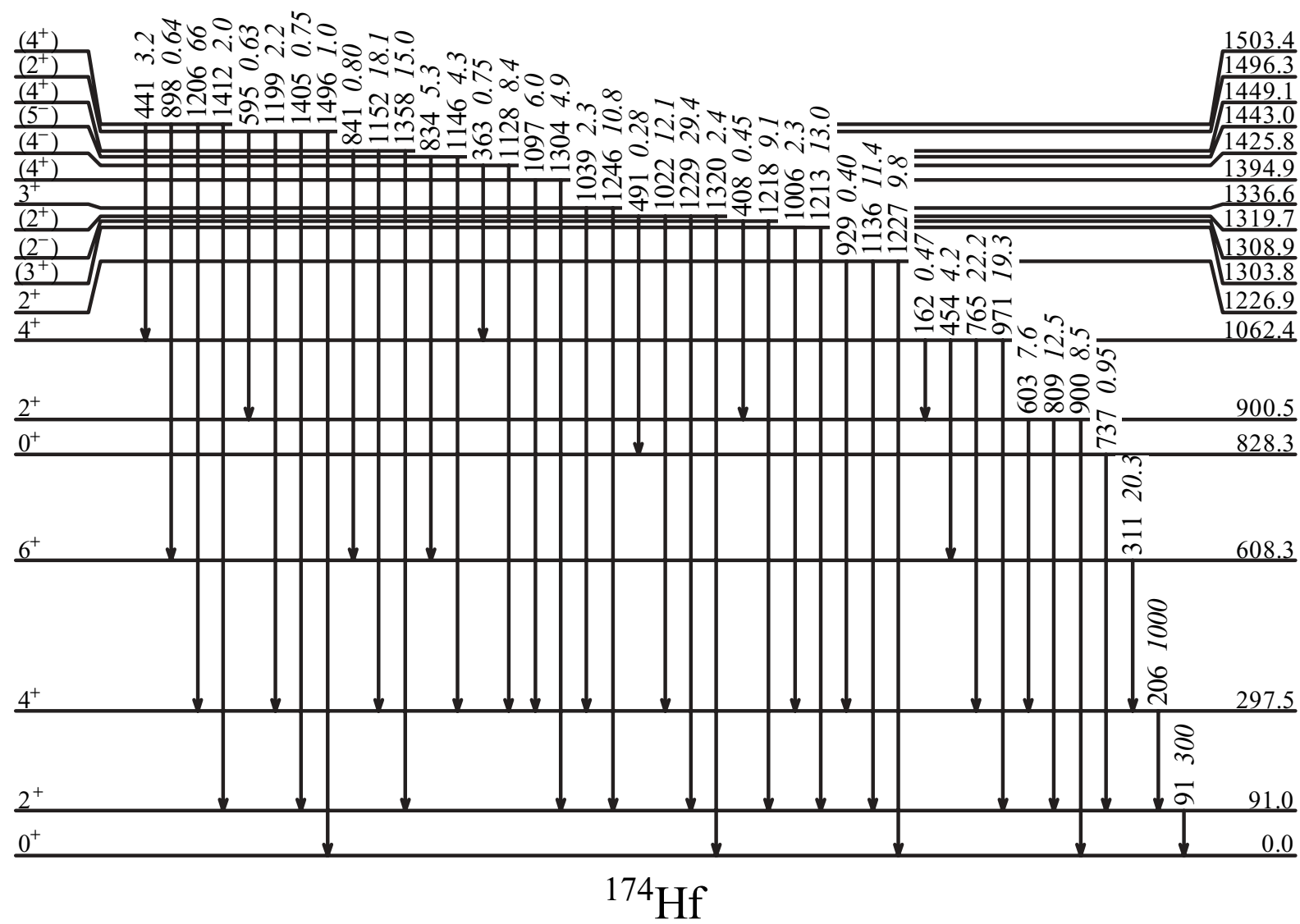

FIG. 12. Level scheme for ${ }^{174} \mathrm{Hf}$ populated in the $\beta^{+} / \epsilon$ decay of ${ }^{174} \mathrm{Ta}$ for levels below $\sim 1500 \mathrm{keV}$. Levels are marked with their energy in keV. $\gamma$-ray transitions are labeled by their energy in $\mathrm{keV}$ and relative intensities (in italics), normalized to the $206-\mathrm{keV}, 4_{1}^{+} \rightarrow 22_{1}^{+}$transition $\left(I_{206} \equiv 1000\right)$. 

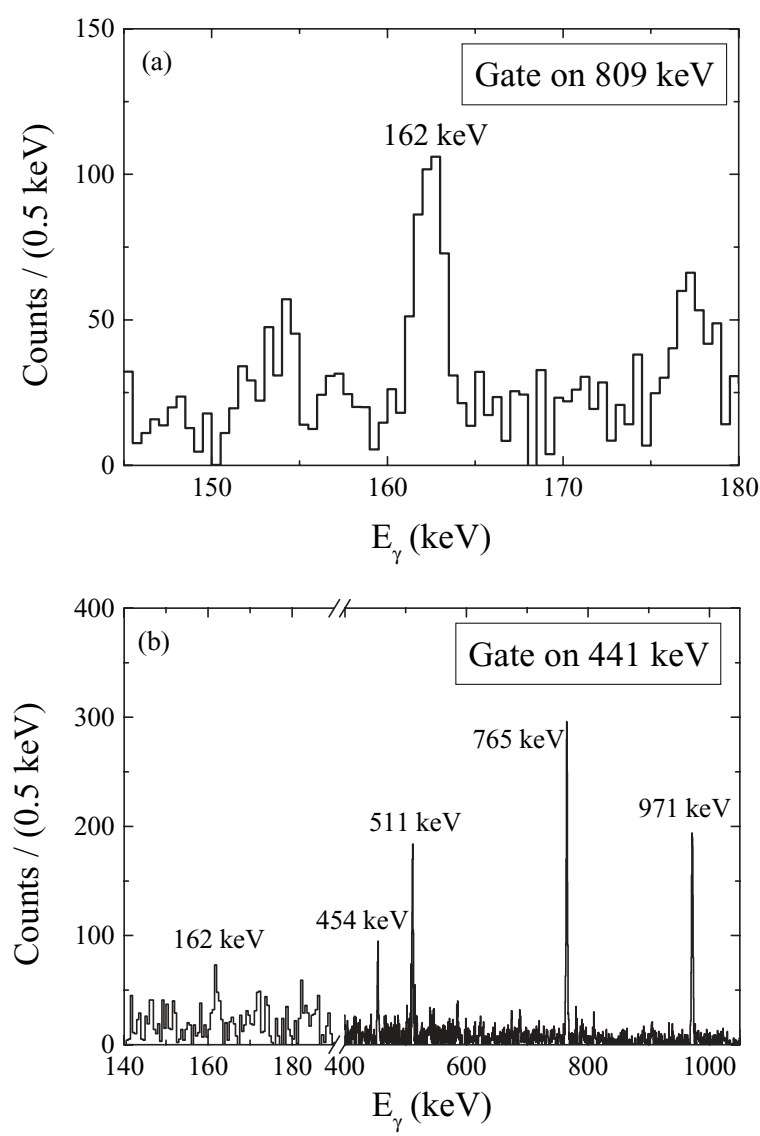

FIG. 13. Spectra providing evidence for the placement of a newly observed $162-\mathrm{keV}$ transition in ${ }^{174} \mathrm{Hf}$. (a) Spectrum gated on the $809-\mathrm{keV}, 2_{2}^{+} \rightarrow 2_{1}^{+}$transition. (b) Spectrum gated on the $441-\mathrm{keV}$ transition that populates the level at $1062 \mathrm{keV}$.

$\gamma$ state, $\epsilon_{\beta \gamma}^{\prime}$ is related to $Z_{\beta \gamma}$ by [3]

$$
Z_{\beta \gamma}=\frac{\sqrt{6}}{\sqrt{24}} \frac{Q_{\beta}}{Q_{\gamma}} \epsilon_{\beta \gamma}^{\prime}
$$

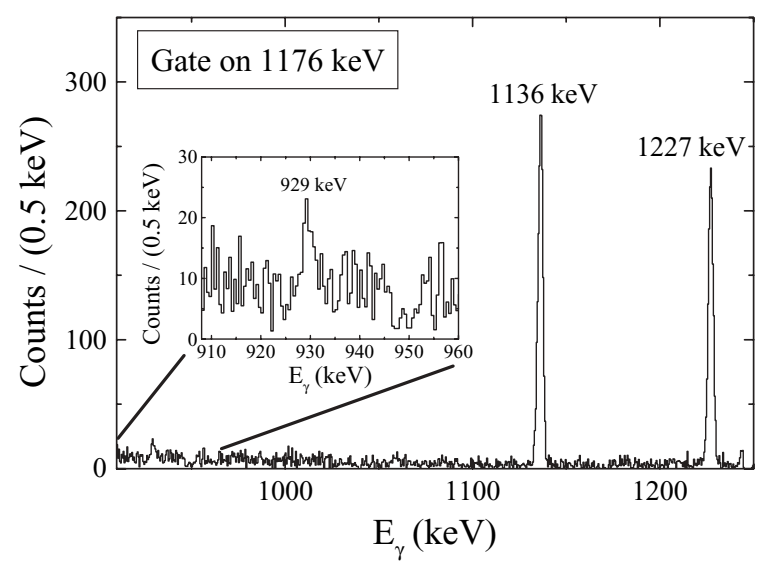

FIG. 14. Spectrum gated on the $1176-\mathrm{keV}$ transition that populates the level at $1227 \mathrm{keV}$ in ${ }^{174} \mathrm{Hf}$. Coincidences are observed with the previously reported 1136 - and $1227-\mathrm{keV}$ depopulating transitions as well as a newly observed $929-\mathrm{keV}$ depopulating transition.

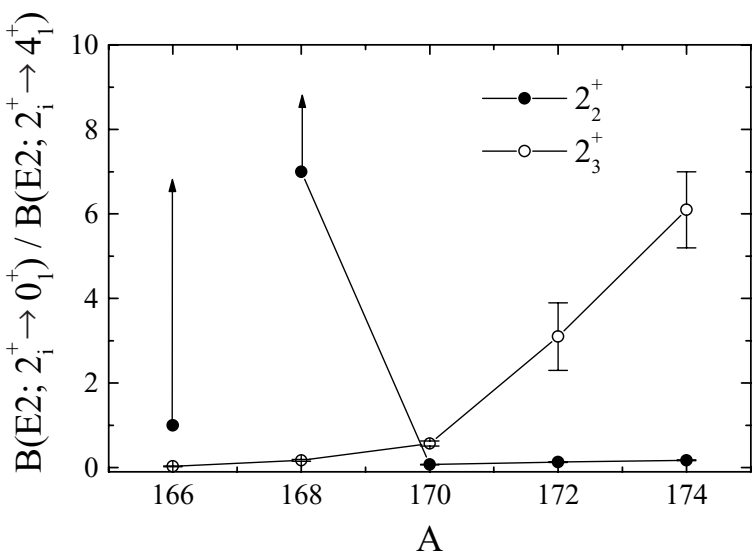

FIG. 15. Branching ratio $B\left(E 2 ; 2_{i}^{+} \rightarrow 0_{1}^{+}\right) / B\left(E 2 ; 2_{i}^{+} \rightarrow 4_{1}^{+}\right)$ from excited $2^{+}$states in the $\mathrm{Hf}$ nuclei. The points for $2_{2}^{+}$in ${ }^{166} \mathrm{Hf}$ and ${ }^{168} \mathrm{Hf}$ are lower limits due to the nonobservation of the $2_{2}^{+} \rightarrow 4_{1}^{+}$ transition (reported with an upper limit on intensity).

where $Q_{\beta(\gamma)}=\sqrt{B\left(E 2 ; 2_{\beta(\gamma)}^{+} \rightarrow 0_{1}^{+}\right)}$. These values have been measured in ${ }^{174} \mathrm{Hf}[18]$, with $B\left(E 2 ; 2_{\gamma}^{+} \rightarrow 0_{1}^{+}\right)=4.8(22)$ W.u. and $B\left(E 2 ; 2_{\beta}^{+} \rightarrow 0_{1}^{+}\right)=2.1(6)$. Taking these as representative values also for the neighboring Hf nuclei gives $\epsilon_{\beta \gamma}^{\prime} \sim 3 Z_{\beta \gamma}$.

In the limit of small mixing, the mixing amplitude (amplitude of unperturbed $\beta$ state in $\gamma$ state) can be written as

$$
\epsilon_{\beta \gamma}^{\prime}(J) \sim \frac{\left\langle\phi_{\beta}(J)\left|V_{\Delta K=2}\right| \phi_{\gamma}(J)\right\rangle}{\left[E_{\gamma}(J)-E_{\beta}(J)\right]_{\text {unp }}} \equiv \frac{V_{\gamma \beta}}{\Delta E_{\gamma \beta}},
$$

where for the sake of simplicity, we have neglected the difference between perturbed and unperturbed spacings. Using Eqs. (4) and (5) along with an average of the $Z_{\beta \gamma}$ values given in Table IV, we obtain the following values for the absolute mixing matrix element between the $2_{\beta}^{+}$and $2_{\gamma}^{+}$excitations:

$$
\begin{aligned}
& { }^{168} \mathrm{Hf}: V_{\gamma \beta} \sim 40 \mathrm{keV} \\
& { }^{172} \mathrm{Hf}: V_{\gamma \beta} \sim 30 \mathrm{keV} \\
& { }^{174} \mathrm{Hf}: V_{\gamma \beta} \sim 35 \mathrm{keV},
\end{aligned}
$$

which are approximately constant across the isotopes but an order of magnitude larger than those extracted in the neighboring $\mathrm{W}$ isotopes.

We note that in the above analysis, we have assumed that the perturbation to the experimental $B(E 2)$ branching ratios arises from mixing only with the ground and $\beta$ bands. In fact, a better determination of $Z_{\beta \gamma}$ should include additional mixing from higher-lying states, not solely the $2_{\beta}^{+}$excitation. However, because these additional states are well separated from the $2_{\gamma}^{+}$excitation, they most likely contribute minimally to the measured $V_{\gamma \beta}$.

To investigate the perturbation, if any, to the energies of excited states, we consider a few energy ratios that provide characteristic indicators of structure, given in Table $\mathrm{V}$. Included are $R_{4 / 2} \equiv E\left(4_{1}^{+}\right) / E\left(2_{1}^{+}\right)$for the ground-state band and the first excited $0^{+}$band as well as the $R 2 \equiv$ $\left[E\left(2_{\beta}^{+}\right)-E\left(0_{2}^{+}\right)\right] / E\left(2_{1}^{+}\right)$ratio. With increasing neutron number, the ground-state $R_{4 / 2}$ values for the $\mathrm{Hf}$ isotopes given in Table V increase and approach the rigid rotor limit of 3.33. 
TABLE V. Energy ratios in the Hf isotopes. Included are the $R_{4 / 2}$ in the ground-state band (g.s.) and the excited $0^{+}$band $\left(0_{2}^{+}\right)$as well as the $R 2 \equiv$ $\left[E\left(2_{\beta}^{+}\right)-E\left(0_{2}^{+}\right)\right] / E\left(2_{1}^{+}\right)$ratio.

\begin{tabular}{lccc}
\hline \hline & $R_{4 / 2}$ (g.s.) & $R_{4 / 2}\left(0_{2}^{+}\right)$ & $R 2$ \\
\hline${ }^{166} \mathrm{Hf}$ & 2.97 & & 0.96 \\
${ }^{168} \mathrm{Hf}$ & 3.11 & 2.94 & 0.94 \\
${ }^{170} \mathrm{Hf}$ & 3.19 & 2.59 & 1.07 \\
${ }^{172} \mathrm{Hf}$ & 3.25 & 3.18 & 0.85 \\
${ }^{174} \mathrm{Hf}$ & 3.27 & 3.24 & 0.79 \\
\hline \hline
\end{tabular}

In all cases, except ${ }^{170} \mathrm{Hf}$, the $R_{4 / 2}$ in the excited $0^{+}$band is slightly smaller than the $R_{4 / 2}$ of the ground-state band yet also increases with increasing neutron number. The ratio of the relative $2^{+}$to $0^{+}$spacing in the excited $0_{2}^{+}$band and the ground-state band, $R 2$, decreases with increasing neutron number, with the exception of ${ }^{170} \mathrm{Hf}$.

The gradual evolution of most energies in Table V suggests that although there is a large matrix element between the $2_{\beta}^{+}$and $2_{\gamma}^{+}$excitations, it does not significantly perturb the energies. Knowing the final perturbed spacing and now the mixing matrix element, we can quantitatively calculate the original unperturbed spacing. Applying a simple two-level mixing analysis, we find that a $35-\mathrm{keV}$ matrix element for ${ }^{174} \mathrm{Hf}$, for example, gives a $\sim 3 \mathrm{keV}$ energy difference between the perturbed and unperturbed $2_{\gamma}^{+}$and $2_{\beta}^{+}$levels. From the energy ratios given in Table $\mathrm{V}$, it appears that the energies of $2_{\gamma}^{+}$and $2_{\beta}^{+}$in ${ }^{170} \mathrm{Hf}$ are significantly more perturbed compared to the neighboring isotopes. From the analysis in terms of $Z_{\beta \gamma}$, the mixing between $2^{+}$states in ${ }^{170} \mathrm{Hf}$ is significantly larger.
As an approximation, we can assume that the $2_{\gamma}^{+}$and $2_{\beta}^{+}$levels were degenerate in their unperturbed location. Taking both the $2_{\gamma}^{+}$and $2_{\beta}^{+}$levels at $975 \mathrm{keV}$ and recalculating the $R_{4 / 2}$ and $R 2$ ratios gives $R_{4 / 2}\left(0_{2}^{+}\right)=2.94$ and $R 2=0.94$. These values now fit within the systematics observed in Table V. Thus, from both the observed branching ratios, and the perturbation to energies, ${ }^{170} \mathrm{Hf}$ appears to be a nucleus where the $2_{\gamma}^{+}$and $2_{\beta}^{+}$ levels are completely mixed.

\section{CONCLUSIONS}

Off-beam $\gamma$-ray spectroscopy was performed on $170,172,174 \mathrm{Hf}$ populated in $\beta^{+} / \epsilon$ decay. New coincidence data yielded significantly revised level schemes for ${ }^{170,172} \mathrm{Hf}$ and confirmed the previous level scheme for ${ }^{174} \mathrm{Hf}$. A simple, two-band mixing formalism was applied to ${ }^{168} \mathrm{Hf}$ through ${ }^{174} \mathrm{Hf}$. The results consistently indicate an absolute mixing matrix element between the $2_{\gamma}^{+}$and $2_{\beta}^{+}$levels on the order of $\sim 30 \mathrm{keV}$. These values are an order of magnitude larger than has been thought in the past. In the case of ${ }^{170} \mathrm{Hf}$, the $2_{\gamma}^{+}$and $2_{\beta}^{+}$level are found to be completely mixed.

\section{ACKNOWLEDGMENTS}

Valuable discussions with C. J. Lister are acknowledged. This work was supported by US DOE grants DE-FG0291ER-40609 and DE-FG02-88ER40417, by the DOE Office of Nuclear Physics under contract DE-AC02-06CH11357, and by the Turkish Atomic Energy Authority under project no. OUK 120100-4.
[1] A. Bohr and B. R. Mottelson, Nuclear Structure (Benjamin, New York, 1975), Vol. II.

[2] G. Alaga, K. Alder, A. Bohr, and B. R. Mottelson, Mat. Fys. Medd. K. Dan. Vidensk. Selsk. 29, 9 (1955).

[3] P. O. Lipas, Nucl. Phys. 39, 468 (1962).

[4] B. R. Mottelson, J. Phys. Soc. Jpn. Suppl. 24, 87 (1968).

[5] L. L. Riedinger, N. R. Johnson, and J. H. Hamilton, Phys. Rev. 179, 1214 (1969).

[6] J. H. Hamilton, F. E. Coffman, A. V. Ramayya, and K. Baker, Phys. Rev. C 3, 960 (1971).

[7] C. Gunther, P. Kleinheinz, R. F. Casten, and B. Elbek, Nucl. Phys. A172, 273 (1971).

[8] P. O. Hess, J. Maruhn, and W. Greiner, J. Phys. G 7, 737 (1981).

[9] C. Y. Wu, D. Cline, E. G. Vogt, W. J. Kernan, T. Czosnyka, A. Kavka, and R. M. Diamond, Phys. Rev. C 40, R3 (1989).

[10] M. Veskovic, W. D. Hamilton, and Krishna Kumar, Phys. Rev. C 41, R1 (1990).

[11] T. L. Khoo, F. M. Bernthal, R. G. H. Robertson, and R. A. Warner, Phys. Rev. Lett. 37, 823 (1976).

[12] P. M. Walker, G. D. Dracoulis, A. Johnston, and J. R. Leigh, Nucl. Phys. A293, 481 (1977).

[13] P. M. Walker, Phys. Scr., T 5, 29 (1983).

[14] E. A. McCutchan, N. V. Zamfir, R. F. Casten, M. A. Caprio, H. Ai, H. Amro, C. W. Beausang, A. A. Hecht, D. A. Meyer, and J. J. Ressler, Phys. Rev. C 71, 024309 (2005).
[15] E. A. McCutchan, R. F. Casten, V. Werner, A. Wolf, Z. Berant, R. J. Casperson, A. Heinz, R. Lüttke, B. Shoraka, J. R. Terry, E. Williams, and R. Winkler, Phys. Rev. C 76, 064307 (2007).

[16] Coral M. Baglin, Nucl. Data Sheets 96, 611 (2002).

[17] Balraj Singh, Nucl. Data Sheets 75, 199 (1995).

[18] E. Browne and Huo Junde, Nucl. Data Sheets 87, 15 (1999).

[19] N. Pietralla, T. Mizusaki, P. von Brentano, R. V. Jolos, T. Otsuka, and V. Werner, Phys. Rev. C 57, 150 (1998).

[20] A. Wolf et al., Phys. Rev. C 76, 047308 (2007).

[21] C. W. Beausang et al., Nucl. Instrum. Methods A 452, 431 (2000).

[22] R. E. Leber, P. E. Haustein, and I.-M. Ladenbauer-Bellis, J. Inorg. Nucl. Chem. 38, 951 (1976).

[23] M. H. Cardoso, P. F. A. Goudsmit, and J. Konijn, Nucl. Phys. A205, 121 (1973).

[24] W. W. Bowman and T. T. Sugihara, Nucl. Phys. A178, 263 (1971).

[25] A. Charvet, Do Huu Phuoc, R. Duffait, A. Emsallem, and R. Chery, J. Phys. (Paris) 32, 359 (1971).

[26] M. T. Gillin and N. F. Peek, Phys. Rev. C 4, 1334 (1971).

[27] M. H. Cardoso, L. Klieb, P. F. A. Goudsmit, and J. Konijn, Z. Phys. A 272, 13 (1975). 